Article

\title{
A Framework of Structural Damage Detection for Civil Structures Using Fast Fourier Transform and Deep Convolutional Neural Networks
}

\author{
Yingying He ${ }^{1}$, Hongyang Chen ${ }^{1}$, Die Liu ${ }^{2}$ and Likai Zhang ${ }^{3, *}$ \\ 1 School of Computer Engineering, Chongqing College of Humanities, Science \& Technology, \\ Chongqing 401524, China; heyingyg@163.com (Y.H.); 15223091504@163.com (H.C.) \\ 2 School of Business, Chongqing College of Humanities, Science \& Technology, Chongqing 401524, China; \\ liudie111@163.com \\ 3 School of Civil Engineering, Chongqing University, Chongqing 400045, China \\ * Correspondence: zhanglikai@cqu.edu.cn
}

Citation: He, Y.; Chen, H.; Liu, D.;

Zhang, L. A Framework of Structural Damage Detection for Civil Structures Using Fast Fourier Transform and Deep Convolutional Neural Networks. Appl. Sci. 2021, 11, 9345. https://doi.org/10.3390/app11199345

Academic Editor: Jean-Jacques Sinou

Received: 12 September 2021

Accepted: 3 October 2021

Published: 8 October 2021

Publisher's Note: MDPI stays neutral with regard to jurisdictional claims in published maps and institutional affiliations.

Copyright: (c) 2021 by the authors. Licensee MDPI, Basel, Switzerland. This article is an open access article distributed under the terms and conditions of the Creative Commons Attribution (CC BY) license (https:// creativecommons.org/licenses/by/ $4.0 /)$.

\begin{abstract}
In the field of structural health monitoring (SHM), vibration-based structural damage detection is an important technology to ensure the safety of civil structures. By taking advantage of deep learning, this study introduces a data-driven structural damage detection method that combines deep convolutional neural networks (DCNN) and fast Fourier transform (FFT). In this method, the structural vibration data are fed into FFT method to acquire frequency information reflecting structural conditions. Then, DCNN is utilized to automatically extract damage features from frequency information to identify structural damage conditions. To verify the effectiveness of the proposed method, FFT-DCNN is carried out on a three-story building structure and ASCE benchmark. The experimental result shows that the proposed method achieves high accuracy, compared with classic machine-learning algorithms such as support vector machine (SVM), random forest (RF), K-Nearest Neighbor (KNN), and eXtreme Gradient boosting (xgboost).
\end{abstract}

Keywords: structural health monitoring; FFT-DCNN; structural damage detection; deep learning; civil structures

\section{Introduction}

Due to environment and load acting on structures, civil structures can produce different levels of damage, such as degradation, corrosion, and fatigue. It reduces the building service life, leading to threats to public safety [1]. Structural health monitoring (SHM) is a critical technology to assure the safety of civil engineering [2,3]. SHM is mainly classified into two categories: data-driven methods based on statistical pattern recognition and physics-based methods based on finite element model updating [4]. Compared with physics-based methods building a numerical model, data-driven methods have many advantages over physics-based methods while identifying structural damage under load and environmental influence such as temperature and moisture effect [5]. Thus, many researchers have focused on data-driven methods for structural damage detection to protect the safety of civil structures. The data-driven method is usually decomposed into several steps: data acquisition, feature extract, damage detection method, and decision-making, where damage detection methods are a big challenge for SHM [6-8].

With the development of computing power, machine learning (ML) algorithms have been widely used in the SHM field. Since vibration signals can reflect structural damage conditions, ML algorithms usually utilize structural vibration data to recognize structural damage, especially in handling vibration data with small datasets $[9,10]$. For example, Seyedpoor [11] adopted a support vector machine (SVM) and differential evolution algorithm (DEA) to identify the structural damage in moment frame connections. Using semi-rigid beam simulated damage in structural connections, and then the vibration data 
of structures with damaged connections were generated by the analytical model. Then, these data were fed into the SVM model to update the model's parameters and weight. The result showed that the SVM-DEA had an excellent performance for structural damage detection. Guo [12] proposed a method based on Bayesian theory and immune genetic algorithm (IGA) to recognize structural damage where Bayesian was applied to identify damage sites, and then improved IGA was utilized to identify the damage level. This study provided a two-stage method that can effectively assess the damage locations and extent. $\mathrm{Wu}$ [13] utilized the parson correlation coefficient to select essential information features, and then the features were fed into Ensemble Generalized Multiclass SupportVector-Machine (EGMSVM) to recognize structural damage. To verify the effectiveness of the proposed method, different algorithms such as LDA, random forest, SVM, was tested on a simulated hydraulic platform. The result showed that the EGMSVM achieved high accuracy and low variance and deviation.

However, these methods, such as support vector machine, ensemble algorithm, Bayesian algorithm, belong to "shallow" machine learning. In addition, although "shallow" ML algorithms recognize structural damage and location with high accuracy for small datasets, it is poor performance in handling the massive vibration data from SHM systems.

With the development of sensors technology and data acquisition, SHM systems can collect an amount of data from various sensors installed on civil structures. Since deep learning methods effectively handle massive data, it has attracted much attention from many researchers in many fields such as image classification $[14,15]$ and natural language processing [16]. In these methods, vibration-based convolutional neural networks (CNN) algorithms are widely utilized in civil engineering since it is powerful in extracting the feature from raw vibration data to recognize structural damage. For example, Nur Sila [17] adopted CNN to capture abstract features and complex classifier boundaries and then classified the damaged and healthy condition of structures. The experimental result showed that $\mathrm{CNN}$ accomplished real-time damage diagnosis and location with high accuracy, robustness, and computational efficiency. Bao [18] proposed a computer vision method that detects structural damage using the $\mathrm{CNN}$ model. The obtained vibration data were transformed into time and frequency domain images via visualization method, and then these images were fed into CNN to classify structural damage. The accuracy of classification can refer to 93.5\%. Abdeljaber [19] and Zhang [20] proposed a one-dimensional CNN with data-processing techniques that utilized a small training sampling to identify structural damage location and mass changes effectively. However, the above methods have some limitations in handling contaminated or noisy data using the $\mathrm{CNN}$ methods because they may regard the contaminated information from acceleration data as fault information. More importantly, contaminated time-sequence data such as acceleration data cannot effectively reflect structural damaged conditions. Thus, many scholars have studied methods based on frequency information. To be specific, time signal is transformed into frequency information, which can reduce the influence from contaminated data and improve the accuracy of damage identification in noisy environments.

For example, Hoshyar [21] converted vibration signal to frequency information and then utilized machine learning algorithms such as support vector machines to localize concrete cracks based on obtained frequency information. The experimental results showed that the proposed method had high accuracy. Tehrani [22] utilized an artificial intelligence method based on fast Fourier transform (FFT) to recognize structural damage degree. The results showed that FFT was suitable for nonstationary vibration signals. Nguyen [23] proposed a method by combining FFT analysis and artificial learning to evaluate damaged changes of structures through the discrete model. The result gained higher accuracy compared with using only other models or analyses. However, the accuracy of FFT combined with traditional methods such as support vector machines needs to be further improved. Although FFT has many advantages in handling time-sequence, the existing methods do not fully consider the advantages of combining FTT with deep learning, such as DCNN for structural damage detection. 
In this paper, high precision and robust structural damage detection method are proposed based on FFT-DCNN. In this method, FFT is utilized to analyze the frequency information, reducing the influence of contaminated data $[24,25]$. DCNN automatically extracts features from structural frequency information [26,27]. Finally, a three-story building structure $[28,29]$ and ASCE benchmark [30] are usually utilized to evaluate the ability of structural damage detection based on our proposed method.

The following are the primary contributions of this paper: (1) A novel sensor datadriven structural damage detection method is proposed by combining FFT with DCNN, which can effectively handle the vibration signal to recognize the structural damage condition. (2) Compared with traditional damage detection such as FFT-SVM and SVM, KNN, random forest, and XGBoost, the experimental result shows that the proposed method achieves higher accuracy damage detection. (3) Since FFT-DCNN takes a short time on test datasets, it indicates that the proposed method can be utilized for the online detection of structural damage conditions in the field of SHM.

The rest of the paper is organized as follows. Section 2 introduces the proposed FFT-DCNN architecture. Section 3 describes the structural damage detection method based on the proposed method. The experimental setup is introduced in Section 4. FFT-DCNN is carried out on a three-story building structure and ASCE benchmark in Sections 5 and 6, respectively. Finally, Section 7 summarizes some conclusions based on FFT-DCNN and potential topics for future research.

\section{Proposed FFT-DCNN Architecture}

Figure 1 illustrates the architecture of the designed FFT-DCNN. It has five layers: input layer, FFT layer, convolutional neural network (Conv1, Conv2, MP1, Conv3, Conv4, and MP2), fully connected network (FC1, FC2), and output layer. In the CNN algorithm, Conv1 represents the first convolutional operation, and MP1 represents the first pooling operation. In this frame, raw vibration data are transformed into frequency information via FFT method. Considering that the $\mathrm{CNN}$ can be powerful in capturing the spatial features, frequency information with one dimension is transformed into a feature map with two dimensions via dimension transformation, improving the ability of features extraction for DCNN. Then, convolutional and max-pooling layers are utilized to capture spatial features from frequency information. Finally, the extracted features are fed into a fully connected network with a "softmax" activation function to recognize the damaged conditions of the structure. In addition, $\mathrm{DCNN}$ is powerful in handling two-dimension data. However, the frequency information data that are transformed by the FFT method is onedimension. DCNN cannot effectively extract the feature from the frequency information. Thus, the dimension transformation concept is referenced to transform one dimension into two dimensions.

\subsection{Fast Fourier Transform Layer}

Fast Fourier transform (FFT), as a discrete Fourier transform algorithm, was first proposed by Cooley and Tukey in the year 1965. It can reduce the number of computations needed for $N$ points from $O\left(N^{2}\right)$ to $O(N \log N)$. With the increasing of sampling points, this method can save more computational resources.

Features extraction of FFT is shown in Figure 2. First of all, utilizing a sliding window acquires $\mathrm{n}$ consecutive time-domain data from the original signal. Then, every window data are transformed frequency information via FFT method, which is described as:

$$
X_{k}=\sum_{n=0}^{N-1} x_{n} e^{-i 2 \pi k n / N} \quad k=0, \ldots, N-1
$$

where $x_{0}, \ldots, x_{N-1}$ is a complex number and $X_{k}$ is time-domain data. $N$ represents the number of sampling points. 


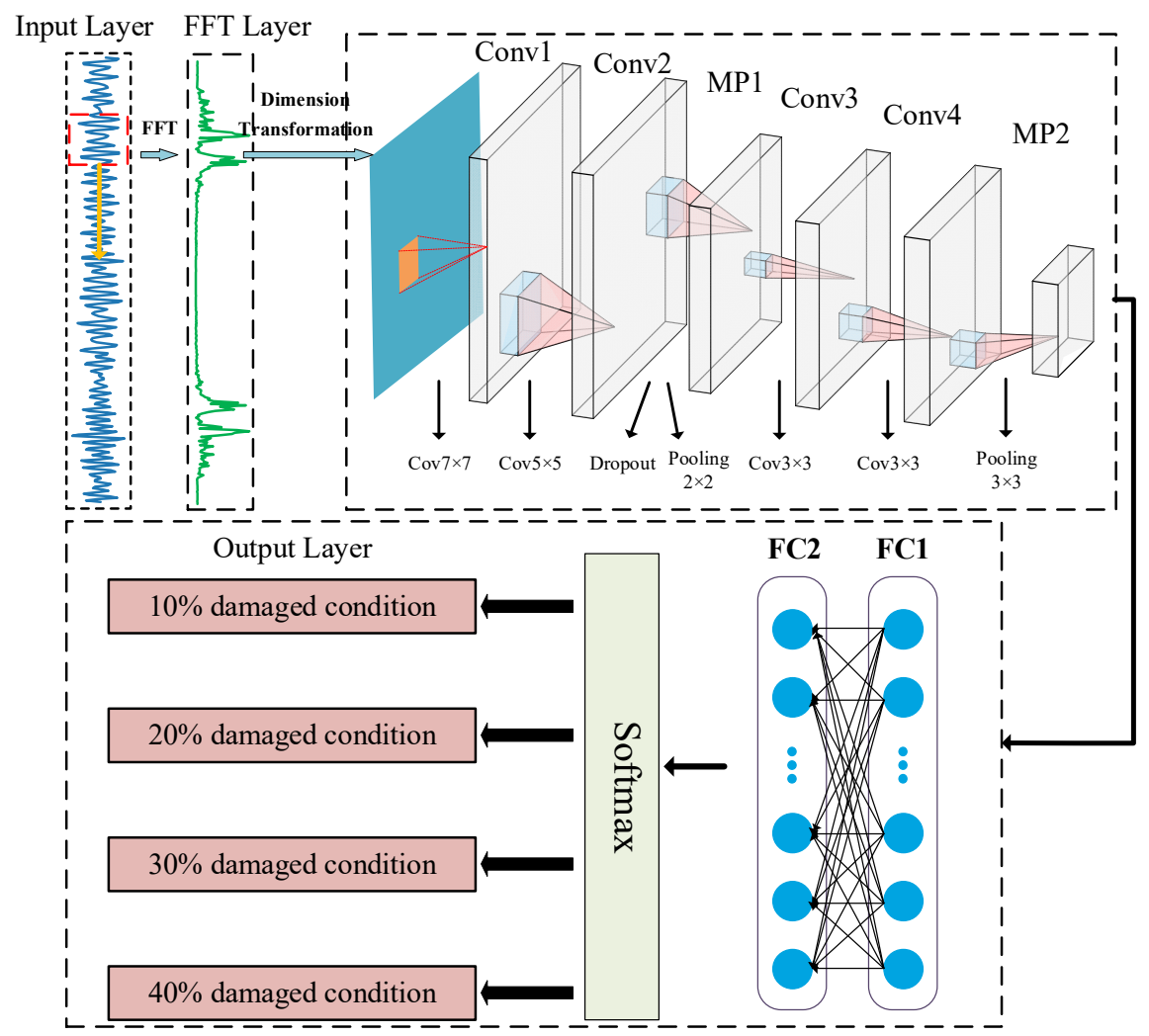

Figure 1. Architecture of the proposed method.

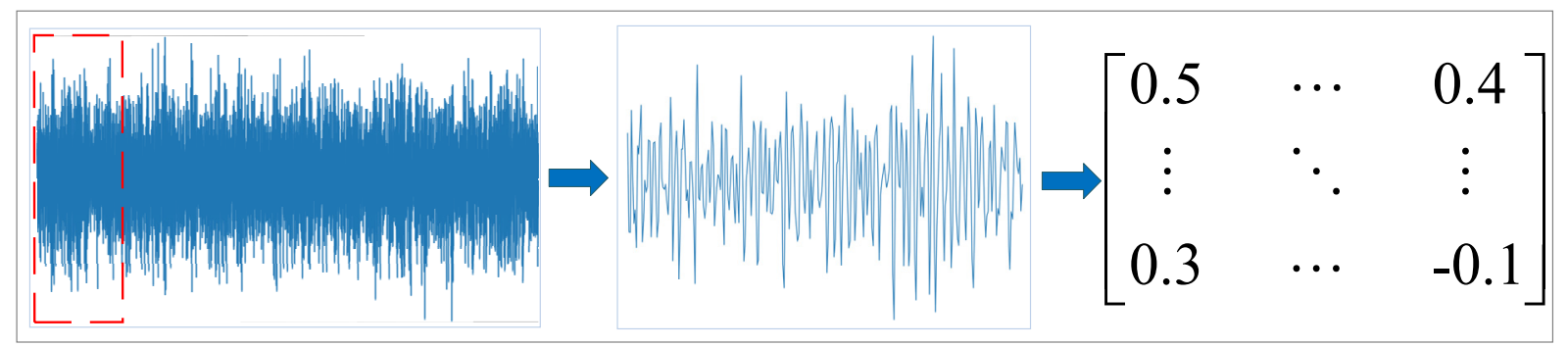

Figure 2. Flowchart of features extraction.

\subsection{Convolutional Layer}

In this study, the DCNN has two main layers, which are a convolutional layer and a pooling layer, respectively. The convolutional layer of DCNN is mainly utilized to extract features from building structures via convolution operation. In this procedure, convolutional kernels slide on the input sample via using the same size convolution kernel $\left(h_{C}, w_{C}\right)$ where $h_{C}, w_{C}$, and $s_{C}$ represent the length, width, and step size of the convolution kernel, respectively. After finishing the convolutional operation, the output matrix can be obtained, and the number of kernels is the same as the output matrix. Output matrix of the convolutional layer is described as:

$$
\begin{aligned}
& C_{j}=f\left(X_{i} * W_{j}+B_{j}\right) \\
& C=\left[C_{1}, C_{2}, \cdots, C_{K_{C}}\right]
\end{aligned}
$$

where $*$ represents the convolutional operation. $W_{j}$ and $B_{j}$ represent the $j$-th $\left(j \in 1,2, \cdots, K_{C}\right)$ convolution kernel and bias, respectively. $f(\cdot)$ denotes activation function. $C_{j}$ and $C$ are the $j$-th output and the entire output, respectively. In addition, the important information 
is lost due to dimensional reduction of input data $X_{i}$, when the input sample $X_{i}$ carries out a convolutional operation leading to a change of the feature dimension. To solve the problem, "same padding" is utilized in this study, which can keep the same dimension between input and output. After the convolutional operation, the dimensional operation of the output result can be described as:

$$
R\left(C_{j}\right)=\left[\frac{n_{l}-h_{C}+2 \times p}{s_{c}}+1\right] \times\left[\frac{m_{l}-w_{C}+2 \times p}{s_{C}}+1\right] \times K_{C}
$$

where $w_{C}$ and $h_{C}$ are length and width of the frequency information via FFT transformation, respectively. $K_{C}$ denotes the number of convolutional kernels. $n_{l}$ and $m_{l}$ are length and width of convolutional kernels. $s_{\mathcal{C}}$ represents the sliding step.

\subsection{Pooling Layer}

After convolution operation, the dimensions and parameters of the input matrix can increase, leading to required computing resources. Thus, a pooling layer is utilized to perform operations of dimension reduction, while it can save the important information of extraction features. In addition, a pooling layer can solve some problems such as overfitting or the long training time. The pooling procedure is described as:

$$
\begin{gathered}
P_{j}=\operatorname{MaxPooling}\left(C_{j}\right) \\
P=\left[P_{1}, P_{2}, \cdots, P_{K_{P}}\right]
\end{gathered}
$$

where $C_{j}$ is convolutional layer and $P_{j}$ presents pooling result of $j$-th convolutional layer. $P$ describe all pooling layers.

\subsection{Fully Connected Layer}

After convolutional and pooling layers, the obtained two-dimensional matrix is transformed into a one-dimensional vector via flattened operation. Then, the vector is fed into a fully connected layer, which is described as:

$$
F=f\left(\sum P \times W_{F}+b\right)
$$

where $P$ represents input vector. $W_{F}$ and $b$ represent weights and bias, respectively. $f(\cdot)$ denotes activation function. $F$ is the outputs of fully connected layers.

\subsection{Classification Layer}

The classification layer adopts the output feature from the fully connected layer to predict different structural damage via a softmax activation function. More specifically, for every input vector $F$, the classification layer can predict the probability of $F$ belonging to different categories. The range of predicted $F$ is between 0 and 1 , and the sum of probability values is 1 . Then $j$-th predicted category is expressed as:

$$
Y_{j}=\frac{\exp \left(F_{j}\right)}{\sum_{j=1}^{n} \exp \left(F_{j}\right)}
$$

where $F_{j}$ represents probability belonging to different categories. $Y_{j}$ is the probability of predicting $j$-th categories via softmax classifier. $n$ represents the number of entire categories.

\section{Structural Damage Detection Method Using Proposed FFT-DCNN Architecture}

This study proposes an FFT-DCNN method where FFT converts one-dimensional vibration signal into frequency information that reflects the structural damage, and the dimension of frequency information is $M \times 1$. Considering that DCNN is powerful for 
extracting spatial features of input data, the frequency information $M \times 1$ is converted to Fre $_{M}=\sqrt{M} \times \sqrt{M}$ the matrix via dimension transformation, which can reflect the spatial information. Then, $\mathrm{Fre}_{M}$ are fed into the DCNN model to distinguish different structural damage condition. Figure 3 shows the specific process of structural damage detection. Firstly, a sliding window is utilized to split the raw acceleration signals to obtain more samples, which is the term of data augmentation means [31]. Then, to make the data dimension transformation more convenient, the sliding window is set to 324 points, considering that the sampling frequency is $322.58 \mathrm{~Hz}$. In addition, a sliding window with a fixed size is utilized to split the raw acceleration signals. Thus, the length of every segment is the same. Frequency information with 324 points can be acquired by utilizing the FFT method, and every segment includes frequency information of acceleration data.

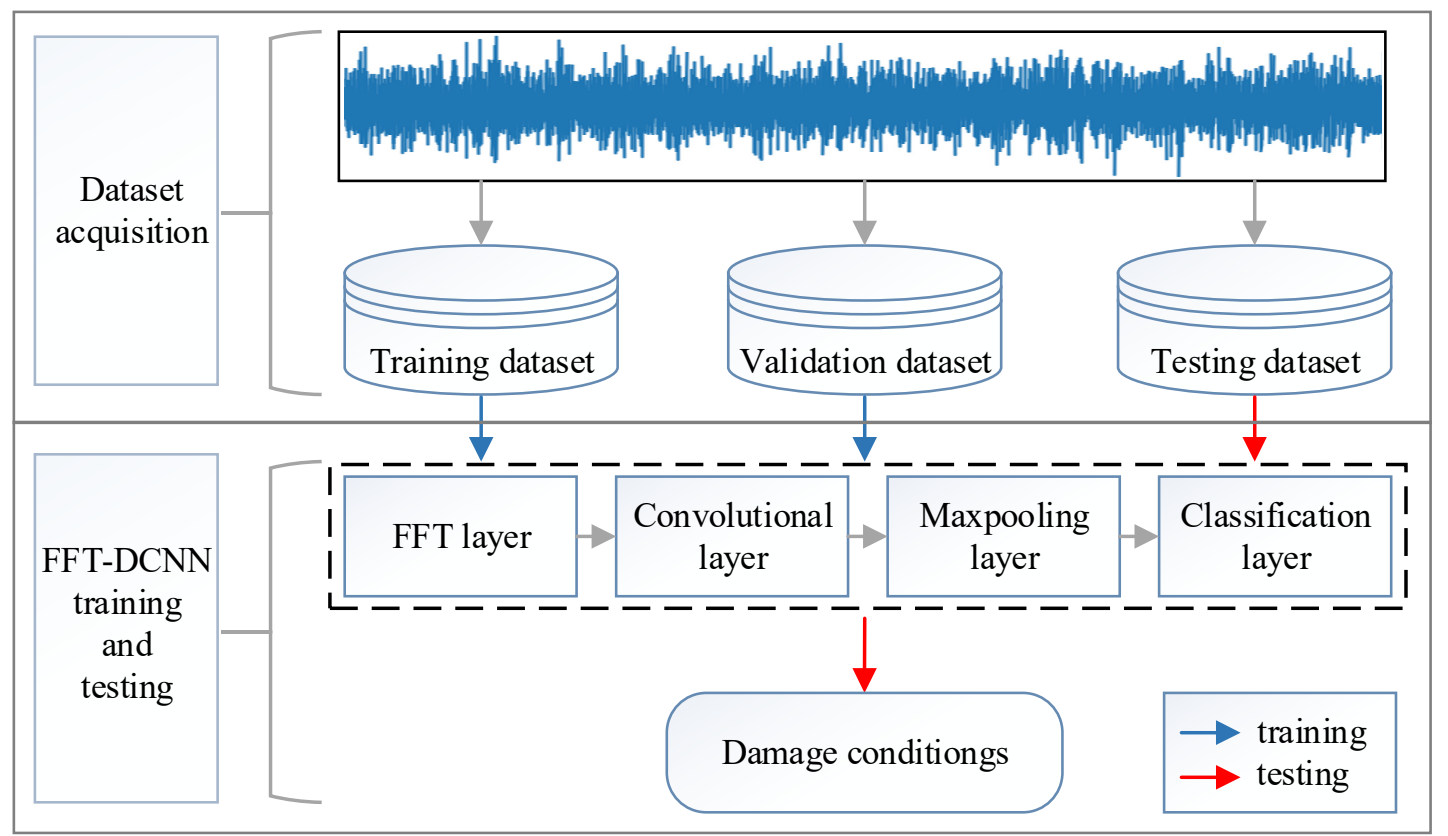

Figure 3. Flowchart of the proposed FFT-DCNN for structural damage detection.

The obtained datasets are randomly divided into training datasets, validation datasets, and testing datasets with a ratio of 6:2:2. Then, the frequency feature matrix $\mathrm{Fre}_{M}$ is then fed into the DCNN model. The loss function and optimizer are used to continuously iterate and update model parameters via training datasets. To reduce the overfitting training process, the model finishes the renewal of model parameters and training process when the training model obtains a preferred result of structural damage detection on verification datasets.

During the training process of FFT-DCNN model, the vibration data are fed into FFT to analyze the frequency information. Then, the $\mathrm{Fre}_{M}$ matrix is fed into convolutional layers extracting spatial features and max-pooling layers reducing trained parameters in the DCNN model. To avoid overfitting problems and improve the ability of features extraction, dropout is added into a fully connected network [32]. After the four convolutional layers and two max-pooling layers extracting features from vibration signals, the features are fed into two fully connected networks with 300, 200 neurons to predict structural damage conditions.

In addition, the optimizer, Adam, is used to update the DCNN model parameters in every iteration. The dropout value is set to 0.5 , and the batch size is set to 512 . The initial learning rate is 0.001 . In this study, the cross-entropy loss function is utilized to assess the training results, which is shown as: 


$$
\text { CrossEntropy }=-\sum_{K=1}^{N}\left(P_{K} \times \log Q_{\mathrm{k}}\right)
$$

where $Q_{\mathrm{k}}$ is the predicted value and $P_{K}$ represents the real value. Finally, after finishing the training, the testing dataset is fed into the DCNN model to identify the degree of structural damage. If the accuracy metric is high, it represents that the FFT-DCNN has excellent performance, and the proposed methods can be applied to actual structural damage detection.

\section{Experimental Setups and Data Description}

This section mainly introduces datasets, experimental settings, and evaluation criteria.

\subsection{Data Description}

Figure 4 shows a three-story building structure is made of aluminum columns and plates assembled using bolted joints with a rigid. The building structure has three floors where the top and bottom of the building $(30.5 \mathrm{~cm} \times 30.5 \mathrm{~cm} \times 2.5 \mathrm{~cm})$ structure are connected by utilizing four aluminum columns $(17.7 \mathrm{~cm} \times 2.5 \mathrm{~cm} \times 0.6 \mathrm{~cm})$ to form a four-degree of freedom system. Moreover, a center column is installed on the top floor, shown in Figure $4 b$, which can change the degree of nonlinearity via changing the distance between the column and the bumper [33]. To collect data from the structure, the structure has five channels, including a force transducer and four acceleration sensors obtaining vibration signals. The sampling frequency is set to $322.58 \mathrm{~Hz}$. Because temperature has a significant influence on the dynamic parameters of the structure monitored, considering that the three-story frame structure was tested in the LOS ALAMOS national laboratory, the temperature should be constant. Thus, the temperature generating influence can be ignored.

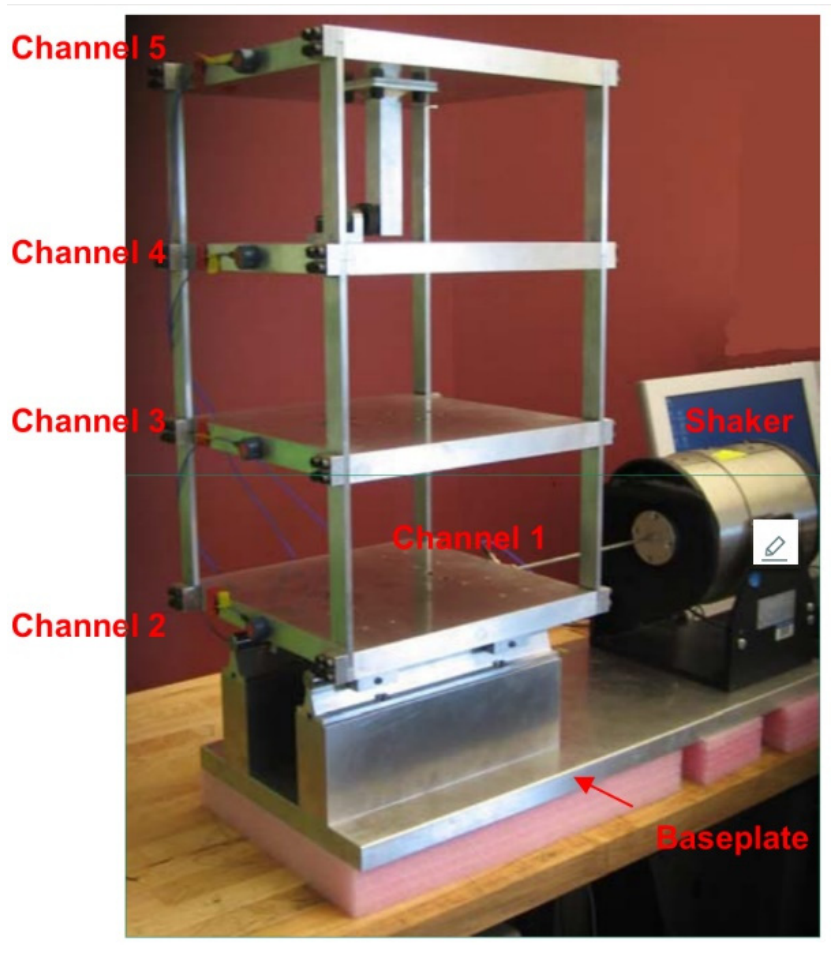

(a) Three-story building structure

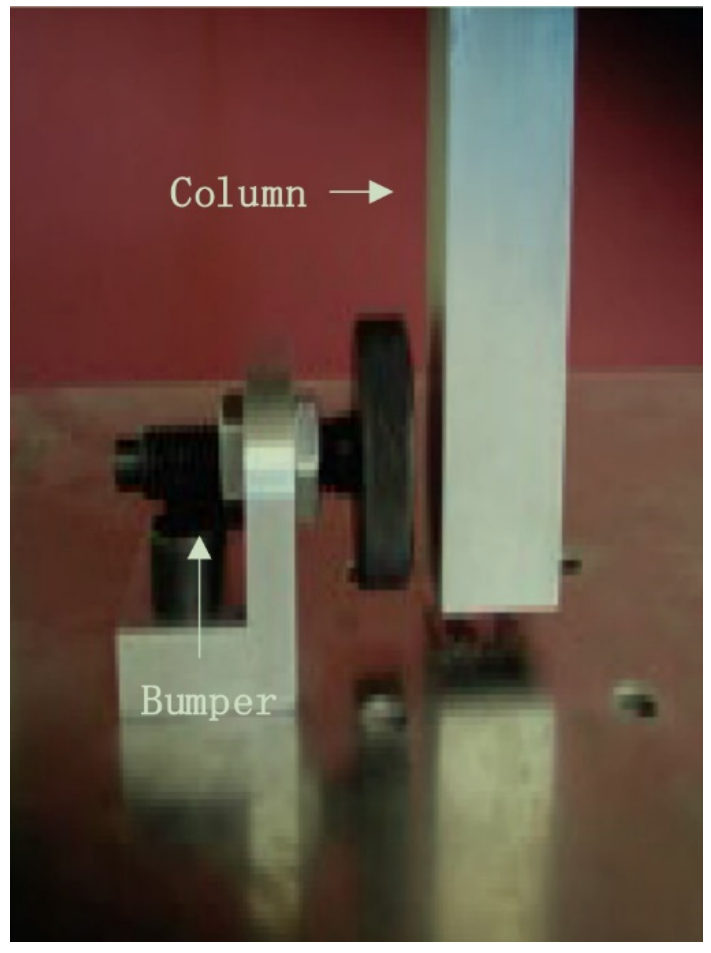

(b) A adjustable bumper

Figure 4. Experimental setup of the building structure. 
According to different damage and nonlinear degree in Table 1, there are some 13 structural conditions. Then, each scenario is repeated ten times, and these data sensors are recorded by sensors. Structural conditions (1-5) represent structural damage caused by nonlinearity, which imitates effects from crack opening and closing. Structural conditions (6-7) are designed as mass and nonlinearity changes, which represent impact-induced damage. Structural conditions (8-13) denote stiffness changes, where structural conditions (11-13) are more severe than (8-10) due to more stiffness reduction of structure.

Table 1. Different scenarios of the structure.

\begin{tabular}{ccc}
\hline Structural Conditions & Label & Description \\
\hline 1 & State\#08 & Gap $=0.13 \mathrm{~mm}$ \\
2 & State\#09 & Gap $=0.10 \mathrm{~mm}$ \\
3 & State\#10 & Gap $=0.05 \mathrm{~mm}$ \\
4 & Gtate\#11 & Gap $=0.15 \mathrm{~mm}$ \\
5 & State\#12 & Gm \\
6 & State\#14 & Gap $=0.20 \mathrm{~mm}+$ mass on the 1st floor \\
7 & State\#15 & Gap $=0.10 \mathrm{~mm}+$ mass on the 1st floor \\
8 & State\#17 & Column: 1BD $-50 \%$ stiffness reduction \\
9 & State\#21 & Column: 3BD $-50 \%$ stiffness reduction \\
10 & State\#24 & Column: 2BD $-50 \%$ stiffness reduction \\
11 & State\#18 & Column: 1AD + 1BD $-50 \%$ stiffness reduction \\
12 & State\#22 & Column: 3AD + 3BD $-50 \%$ stiffness reduction \\
13 & State\#23 & Column: 2AD + 2BD $-50 \%$ stiffness reduction \\
\hline
\end{tabular}

It can be seen from Table 2 that 13 structural conditions can be divided into six scenarios that represent a different degree of structural damage, namely $10 \%$ damage condition, $20 \%$ damage condition, 30\% damage condition, and $40 \%$ damage condition. Then, these four damaged conditions can be divided into the training set, testing set, and validation set with radio 6:2:2. In addition, every state includes 8192 time domain data points. The sampling time is $25.44 \mathrm{~s}$ because the sampling frequency is set to $322.58 \mathrm{~Hz}$.

Table 2. Description of used datasets.

\begin{tabular}{ccc}
\hline Condition Type & State Selection & $\begin{array}{c}\text { Dataset Division } \\
\text { (Training/Validation/Testing) }\end{array}$ \\
\hline 10\% damaged condition & State 08, State 09, State 10, & $3033 / 1012 / 1012$ \\
20\% damaged condition & State 11, State 12 & $1212 / 404 / 404$ \\
$30 \%$ damaged condition & State 17, State 21, State 24 & $1819 / 607 / 606$ \\
$40 \%$ damaged condition & State 18, State 22, State 23 & $1819 / 607 / 606$ \\
\hline
\end{tabular}

\subsection{Crossvalidation for Datasets}

Datasets dividing into the training dataset, validation dataset, and testing dataset with 6:2:2 ratio and the validation are utilized to evaluate the performance of the proposed deep learning algorithm. However, the single split cannot ensure achieving an optimal result of identifying structural damage conditions among subdataset. Therefore, the $K$-fold crossvalidation method is adopted to reduce the bias during the testing process. More specifically, the datasets are divided into the training and testing datasets with a ratio of $8: 2$. Then, the training dataset is split into K equal portions where one portion is validation, and the remaining datasets are training. In this study, the $K=4$ is selected, and the crossvalidation of datasets is shown in Figure 5. 


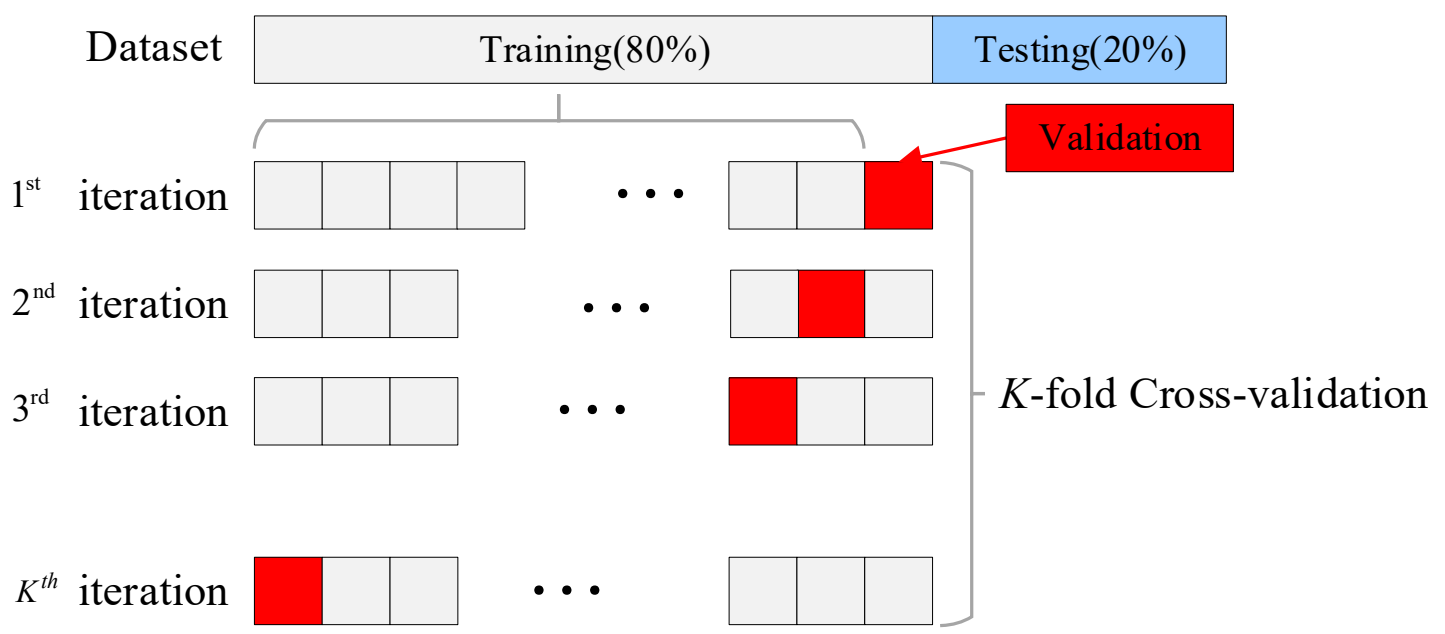

Figure 5. K-fold crossvalidation method.

\subsection{Evaluation Metrics}

Four evaluation metrics, including Accuracy, Precision, Recall, and $\mathrm{F}_{1}$-sore, are selected to evaluate the proposed method and compared algorithms. The above four metrics are defined as follows:

$$
\begin{gathered}
F_{1}=2 \times \frac{\operatorname{Pr} \times \operatorname{Re}}{P r+R e} \\
\operatorname{Recall}(y, \hat{y})=\frac{T P}{T P+F N} \\
\operatorname{Precision}(y, \hat{y})=\frac{T P}{T P+F P} \\
\operatorname{Accuracy}(y, \hat{y})=\frac{T P+T N}{T P+F P+T N+F N}
\end{gathered}
$$

where $y$ and $\hat{y}$ represent the true labels and predicted labels. TP and $F P$ are the number of true positives and are the number of false positives. $T N$ and $F N$ are true negatives and false negatives, respectively.

\section{Experimental Results and Discussion for Three-Story Building Structure}

The section uses a three-story building to evaluate the effectiveness of the proposed method.

\subsection{Experimental Setup and Data Description}

Because the sliding window length is set to 324, every one-dimensional vibration signal with 324 sampling length is obtained. The vibration signal are transformed into frequency information with $324 \times 1$ via FFT method. Then, frequency information is converted to $\mathrm{Fre}_{M}=18 \times 18$ features matrix via shape dimension transformation, considering that DCNN is adopted at extracting spatial features. Finally, DCNN extracts damaged features from $\mathrm{Fre}_{M}$ matrix to identity structural damage. Figure 6 shows the process of features transformation of four different damage condition, including that damaged degree is $10 \%$, damaged degree is $20 \%$, damaged degree is $30 \%$, and damaged degree is $40 \%$. The frequency information of different damage conditions is different with increasing damage degree. 


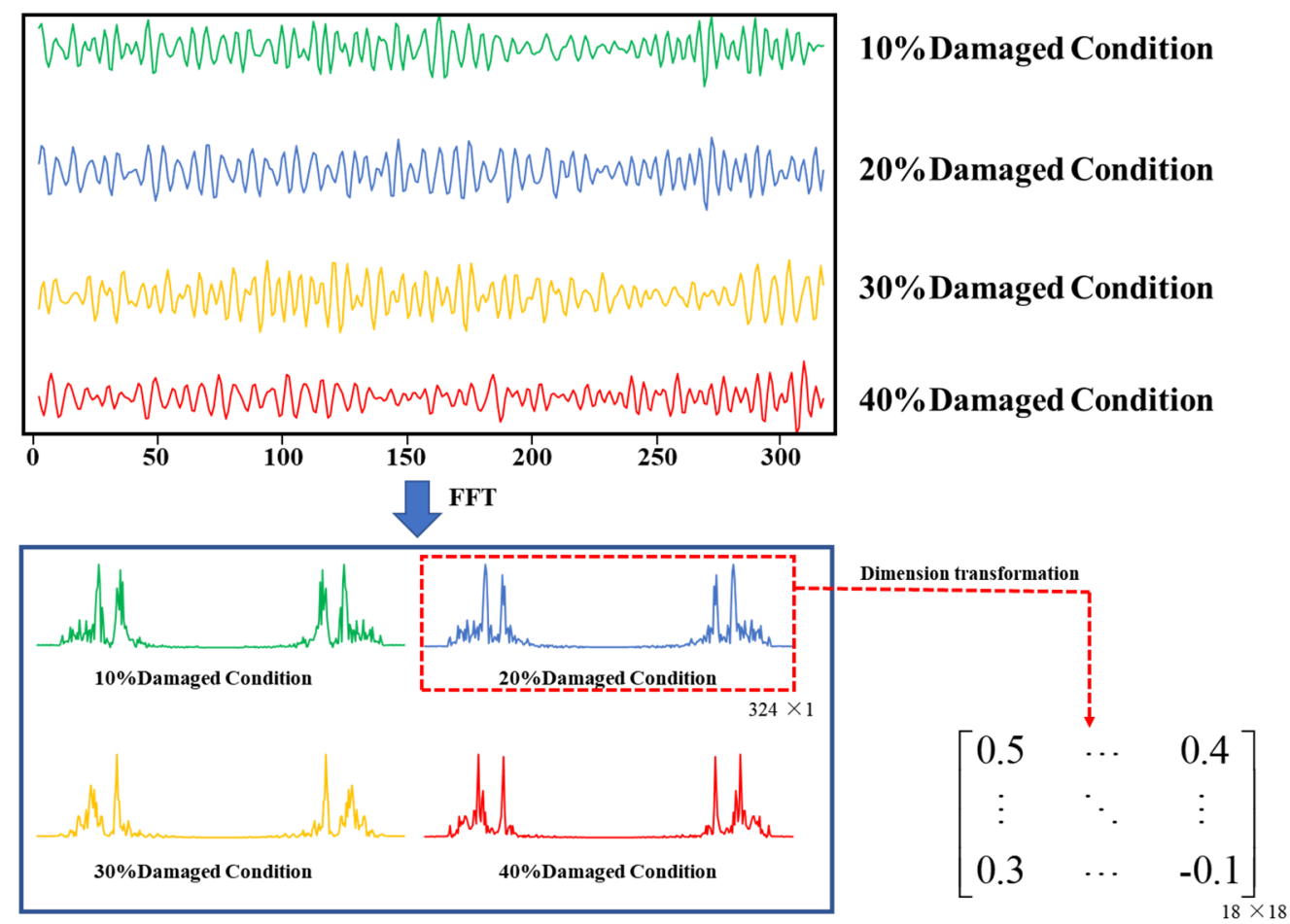

Figure 6. Vibration signal decomposition via FFT method.

Table 3 shows the configuration of the proposed method. The size of the convolutional kernel is $7 \times 7$ in the first layer, and the size of the convolutional kernel is $5 \times 5$ in the second. The maximum pooling layer is $2 \times 2$ in the third layer, the size of the convolutional kernel in the fourth layer is $3 \times 3$ in the fifth layer, and the size of the convolutional kernel is $3 \times 3$ in the sixth layer, and the maximum pooling layer is $2 \times 2$ in the seventh layer.

Table 3. Parameters configuration of DCNN.

\begin{tabular}{ccccccc}
\hline Number & Layer Name & Filter Size & Kernel Size & Stride & Padding & Output Size \\
\hline First layer & Convolution 1 & 8 & $7 \times 7$ & $1 \times 1$ & 1 & $18 \times 18$ \\
Second layer & Convolution 2 & 12 & $5 \times 5$ & $1 \times 1$ & 1 & $18 \times 18$ \\
Third layer & Dropout 1 & - & - & - & - & $18 \times 18$ \\
Fourth layer & Max-pooling 1 & 12 & $2 \times 2$ & $1 \times 1$ & $1 \times 9$ & $9 \times 9$ \\
Fifth layer & Convolution 3 & 16 & $3 \times 3$ & $1 \times 1$ & 1 & $9 \times 9$ \\
Sixth layer & Convolution 4 & 20 & $3 \times 3$ & $1 \times 1$ & 1 & $3 \times 3$ \\
Seventh layer & Max-pooling 2 & 20 & $3 \times 3$ &
\end{tabular}

Table 4 shows the four-fold crossvalidation result on three three-story building structures using the proposed method. In the four-fold crossvalidation, the accuracy of training datasets is $93.48 \%$ on average, and the accuracy of testing datasets is $93.29 \%$ on average. It shows that FFT-DCNN is suitable for identifying structural damage degree effectively.

Table 4. Accuracy of four-fold crossvalidation.

\begin{tabular}{ccc}
\hline Method & Validation $\mathbf{( \% )}$ & Testing (\%) \\
\hline Fold-1 & 93.31 & 93.72 \\
Fold-2 & 93.26 & 92.17 \\
Fold-3 & 93.38 & 93.95 \\
Fold-4 & 93.98 & 93.31 \\
Mean & 93.48 & 93.29 \\
\hline
\end{tabular}


Figure 7 shows the accuracy curve for the proposed methods on Fold- 1 datasets. The accuracy of training, testing, and validation reach 0.9 after achieving 200 epochs. The results show that FFT-DCNN presents an excellent ability of features extraction on the three-story building structure. In Figure 8, the loss curve of the FFT-DCNN model is smooth, which shows an excellent fitting ability and training process.

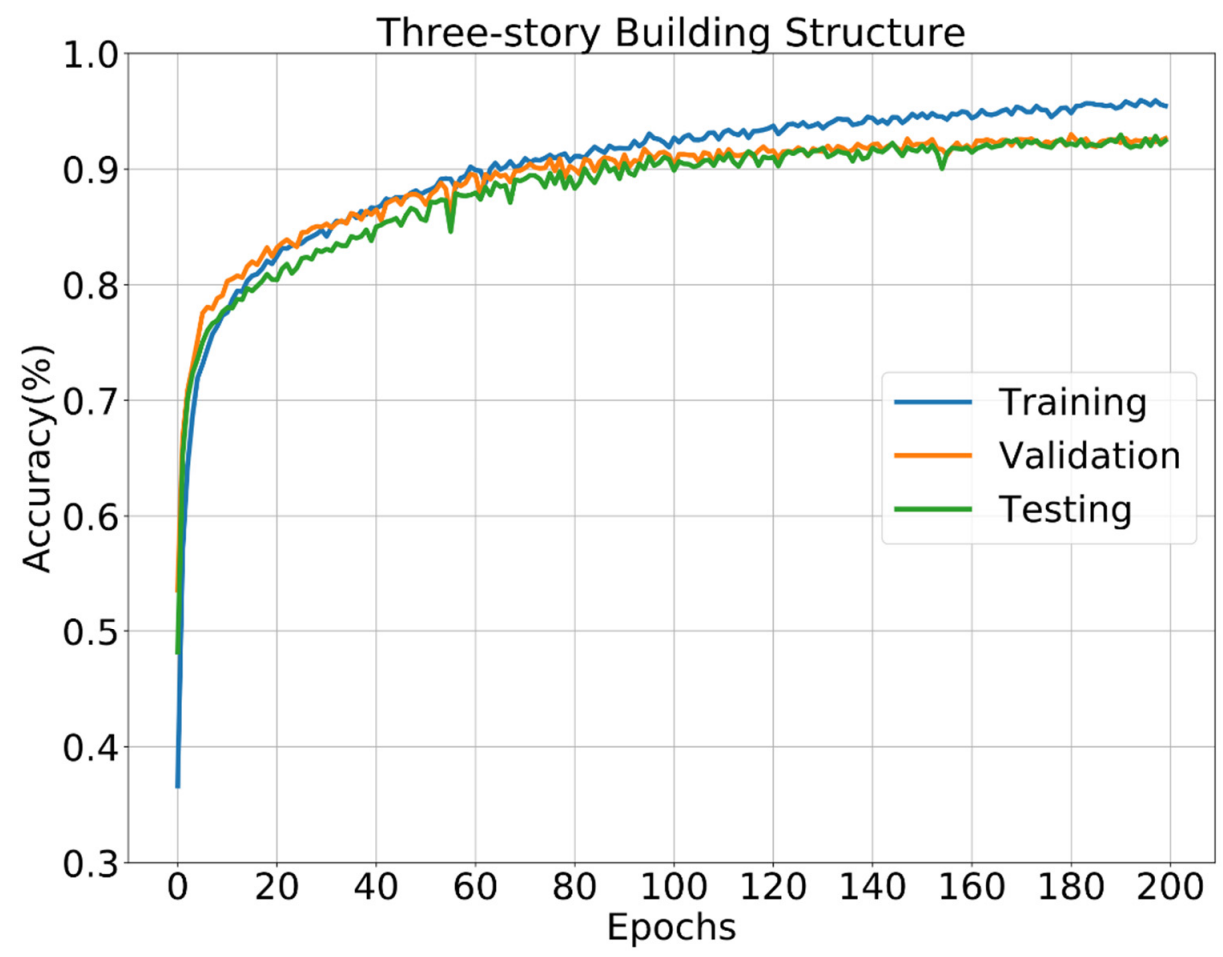

Figure 7. Training accuracy history of the FFT-DCNN algorithm.

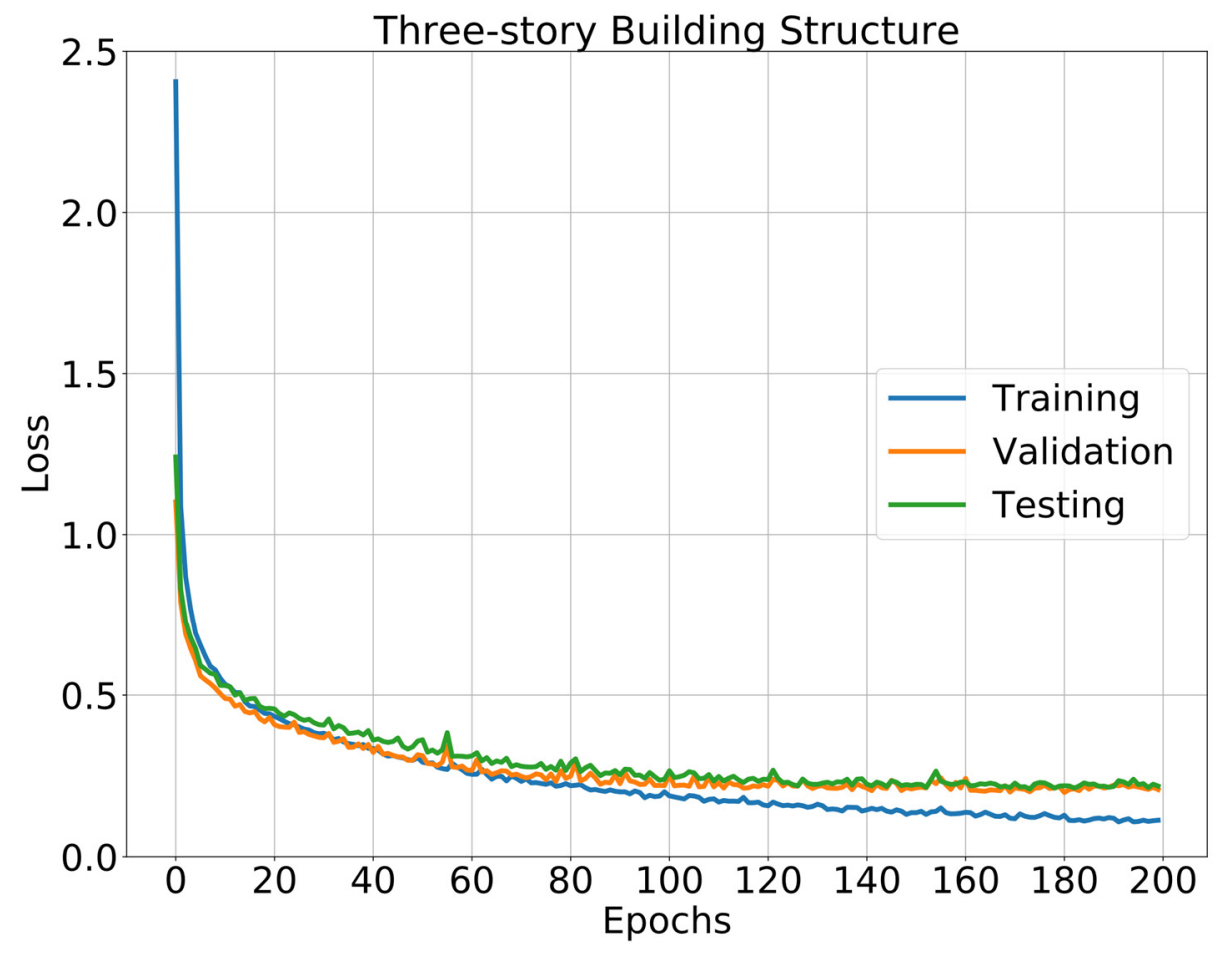

Figure 8. Training loss history of the FFT-DCNN algorithm. 


\subsection{Compared with Other Methods}

To verify the advantages of our proposed method, classical ML algorithms including support vector machine (SVM) [34], FFT-SVM, random forest (RF) [35], K-nearest neighbor (KNN) [36], and eXtreme gradient boosting (XGBoost) [37] are selected to evaluate structural damage degree and improved accuracy in structural damage detection. The accuracy of $\mathrm{KNN}$, RF, and XGBoost is $67.64 \%, 70.24 \%$, and $75.78 \%$, respectively, representing a low ability for recognizing structural damage detection. For these algorithms, such as SVM, $\mathrm{KNN}$, RF, and XGBoost, the time-sequence data of acceleration are input datasets. For FFT-DCNN and FFT-SVM algorithms, the time sequence is transformed into frequency information by the FFT method, and then frequency information is fed into DCNN or SVM algorithms. The relative setting of these algorithms are as follows:

SVM: A Gaussian RBF function is used as the SVR kernel function, and a grid search is used to determine the penalty parameters $c$ and kernel parameters $g$. The search range $c$ and $g$ are $\left[10^{-4}, 10^{4}\right]$ and $\left[2^{-4}, 2^{4}\right]$ by the grid searching method, respectively. Tolerance for stopping criterion is set to $1 \mathrm{e}-3$, and it is enough to satisfy the error criterion.

KNN: The number of neighbors is set to 5 . The value of function weights is set to uniform, representing that all points in each neighborhood are weighted equally. The power parameters. The Minkowski metric is set to 1 , and it is equivalent to using Manhattan distance. The leaf size that affects the construction and query speed is set to 30 .

RF: The number of trees in the forest is 100 , and the maximum depth is set 3 . The min samples split is set to 2, which denotes the minimum number of samples required to split an internal node. Min samples leaf represents that training samples in each of the left and right branches, and the values of Min samples leaf are set to 5. The value of Max features is set to 'auto', representing that the number of features to be considered when looking for the best split.

XGBoost: The maximum depth of a tree is set to 6, and the minimum child weight depth of a tree is 1 . To making the update step more conservative, the max delta step is set to 0.1 . L2 regularization term on weights is set 1 , which can reduce the overfitting problem. The learning rate is set to 0.3 . The fraction columns of random samples for each tree are set to 1 .

Table 5 shows the comparison of damage detection ability between the algorithms mentioned above and the proposed method. It can be seen from Table 5 that the proposed method has higher accuracy in $93.38 \%$ than classical ML methods. In addition, the FFTSVM improves accuracy by $4.56 \%$ than SVM when FFT is added to SVM. It shows that the preprocessing method, for example, FFT method, can reduce the effect of fault or noisy data and improved accuracy in structural damage detection.

Table 5. Comparison result between the proposed method and ML algorithms.

\begin{tabular}{cc}
\hline Method & Accuracy (\%) \\
\hline FFT-DCNN & 93.29 \\
FFT-SVM & 90.15 \\
SVM & 85.59 \\
KNN & 67.64 \\
RF & 70.27 \\
XGBoost & 75.78 \\
\hline
\end{tabular}

To observing the classification result of every defined pattern for test data based on different algorithms, evaluation criteria including Precision, Recall, and $\mathrm{F}_{1}$-sore are utilized in Figures 9-11. Recall represents the number of positive class predictions made out of all positive examples in the dataset. It can be seen from Figure 9 that the obtained Recall scores of $10 \%, 20 \%$, and $30 \%$ damaged conditions are approximately over 0.8 using the FFT-DCNN algorithm, with the exception of $40 \%$ damaged conditions, for which the scores fall approximately 0.5 . In addition, using the precision evaluating on test data, similar results are achieved for FFT-DCNN algorithm. For 10\%, 20\%, 30\%, and 40\% damaged 
conditions, the Precision is approximately over 0.7 with a high recognition performance, which is shown in Figure 10. It can be seen from Figure 11 that the exception of $40 \%$ damaged conditions achieves a low score, and other patterns (such as $10 \%, 20 \%$, and $30 \%$ damaged conditions) obtain a high score using FFT-DCNN algorithm.

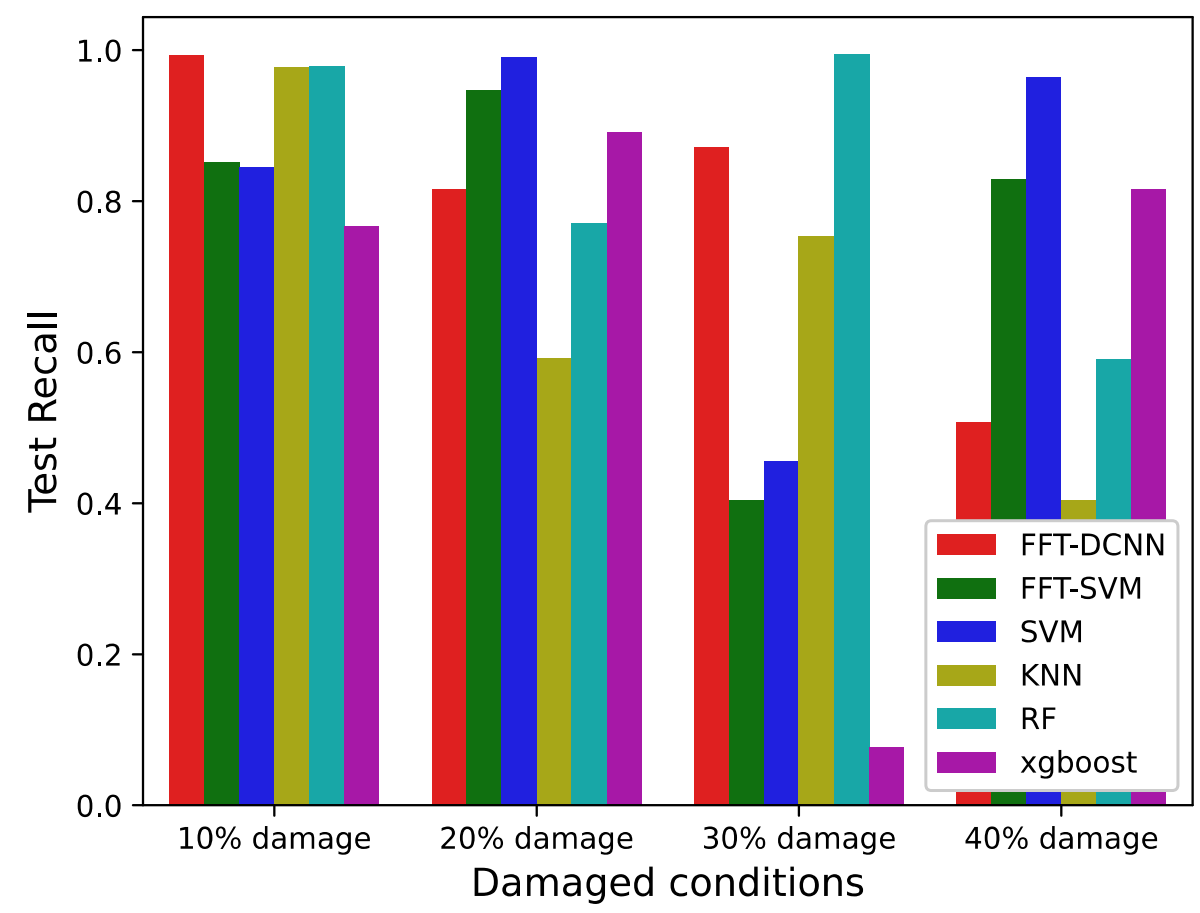

Figure 9. Recall of the test data using different algorithms.

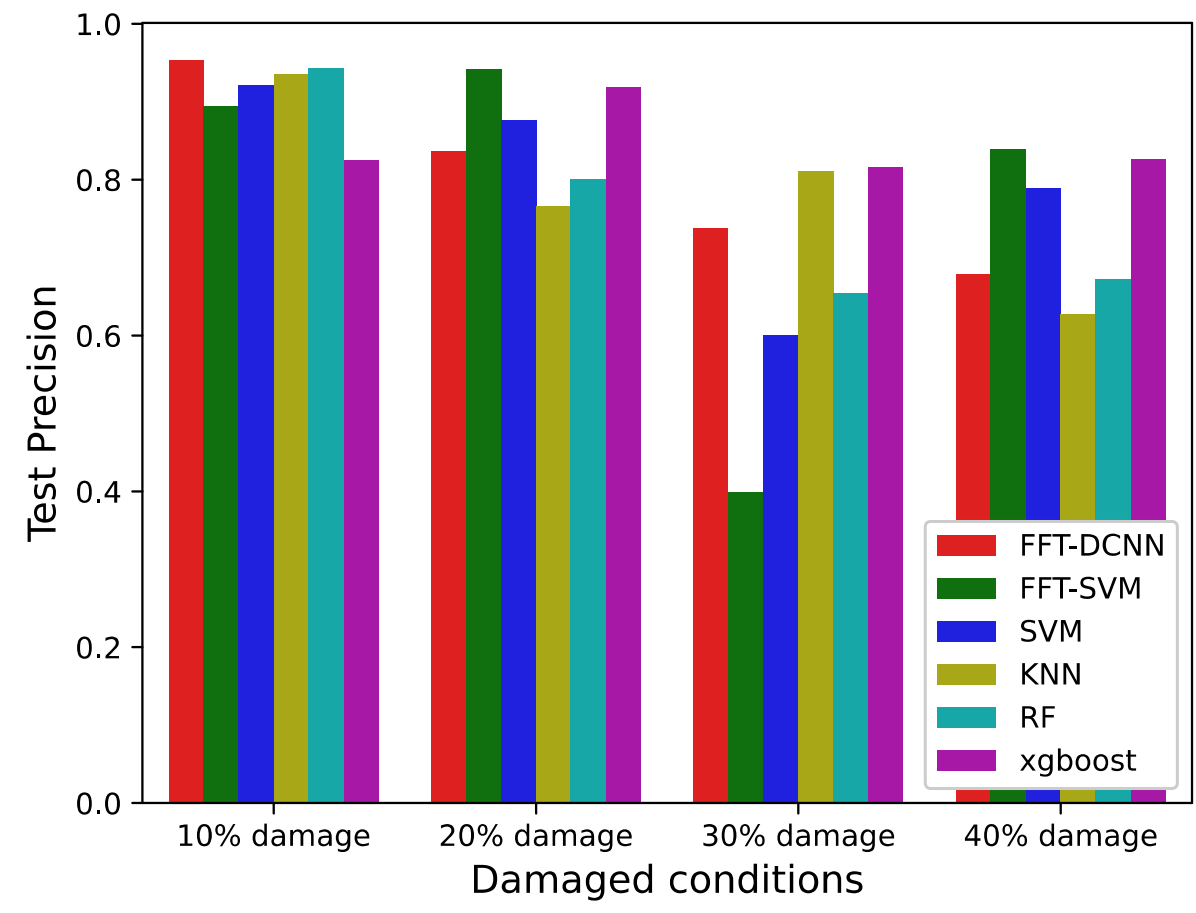

Figure 10. Precision of the test data using different algorithms. 


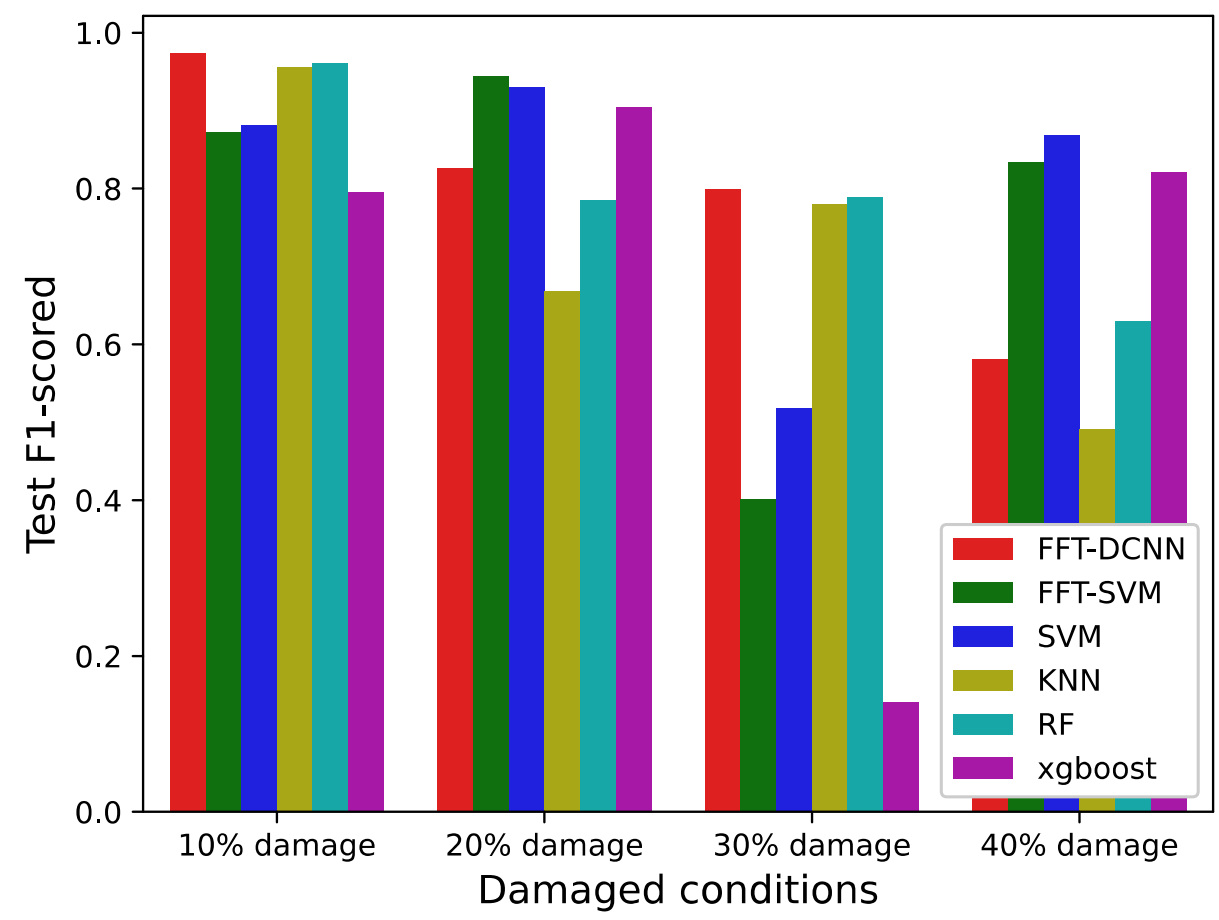

Figure 11. $\mathrm{F}_{1}$-scored of the test data using different algorithms.

In order to reveal the classification of different algorithms under four damaged conditions of a three-story building structure, Figure 12 shows the confusion matrix of the proposed approach and compared algorithms. More specifically, in the FFT-DCNN method, the accuracy of $10 \%$ damaged condition and $40 \%$ damaged condition keep a high value over $95 \%$. For $20 \%$ damaged condition, 37 samples are misclassified as 30\% damaged condition, and 11 samples are misclassified as $10 \%$ damaged condition. The Precision keeps a high value of over $86 \%$. The Precision is $86.7 \%$ for $30 \%$ damaged condition. Finally, the total accuracy of test data for FFT-DCNN is $93.29 \%$, which illustrates the excellent ability of the proposed method for recognizing structural damage conditions compared with other methods, including FFT-SVM (90.15\%), SVM $(85.59 \%)$, KNN (67.64\%), RF $(70.27 \%)$, and XGBoost $(75.78 \%)$. In addition, for the FFT-SVM algorithm, the numbers of classification accuracy in 0-3 damaged conditions are 1003, 239, 468, and 541, respectively, in Figure 12b. The numbers of classification accuracy in 0-3 damaged conditions are 991, 310, 495, and 575, respectively, in Figure 12c. It indicates that accuracy can be improved when the acceleration data are transformed into frequency information. More importantly, the frequency of the three-story frame generates obvious changes compared to methods based on time sequence when the structure suffers different damaged degrees. Our proposed method utilizes frequency information of three-story frame to recognize structural damage with high performance.

All experimental algorithms are performed in the same Windows server, and the server is configured as: GPU is GeForce RTX 3080Ti, RAM is 32 GB, and AMD Ryzen 9 5950X 16-Core Processor. It can be seen from Figures 13 and 14 and Table 6 that the proposed methods cost more time than ML algorithms during training and testing datasets. It is mainly because FFT-CNN continually updates the parameter of the network using a number of datasets. In addition, the training process finishes when the network's loss curve begins to converge, which can cost a large amount of time in Figure 13. With the increase in computing ability, the consuming time of algorithms will be reduced in the future. Thus, the accuracy of structural damage detection should receive the primary attention, which can affect public safety. 


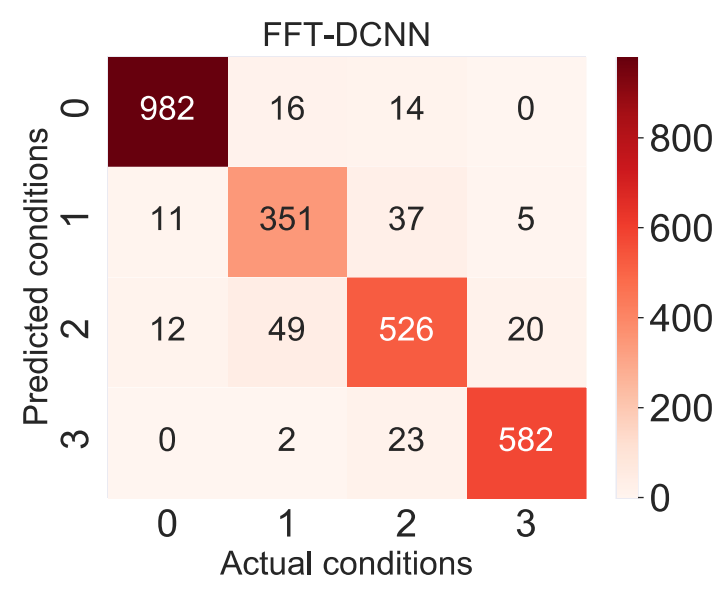

(a) FFT-DCNN confusion matrix

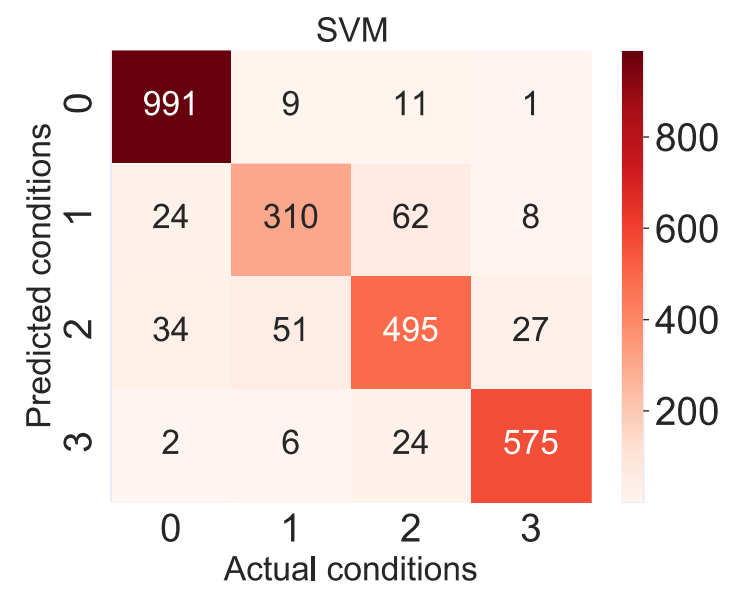

(c) SVM confusion matrix

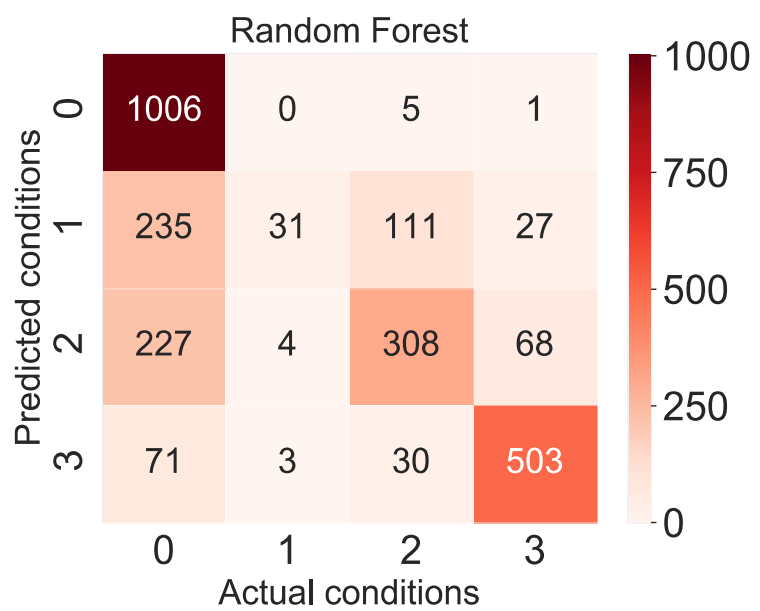

(e) RF confusion matrix

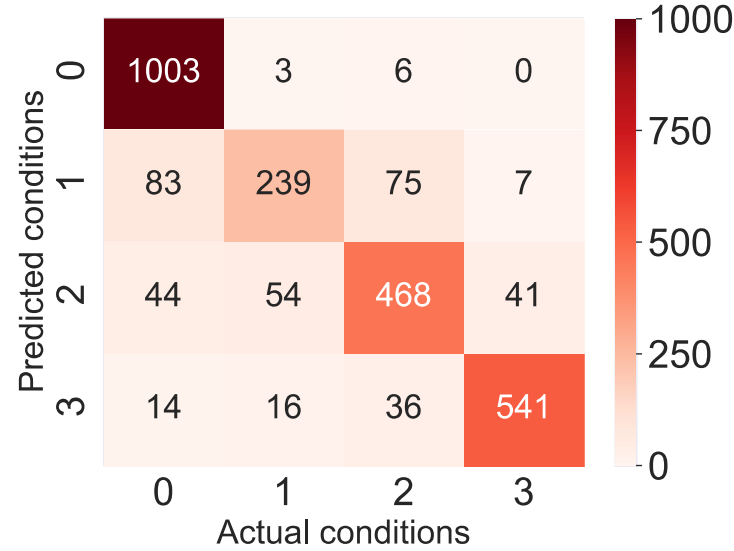

(b) FFT-SVM confusion matrix

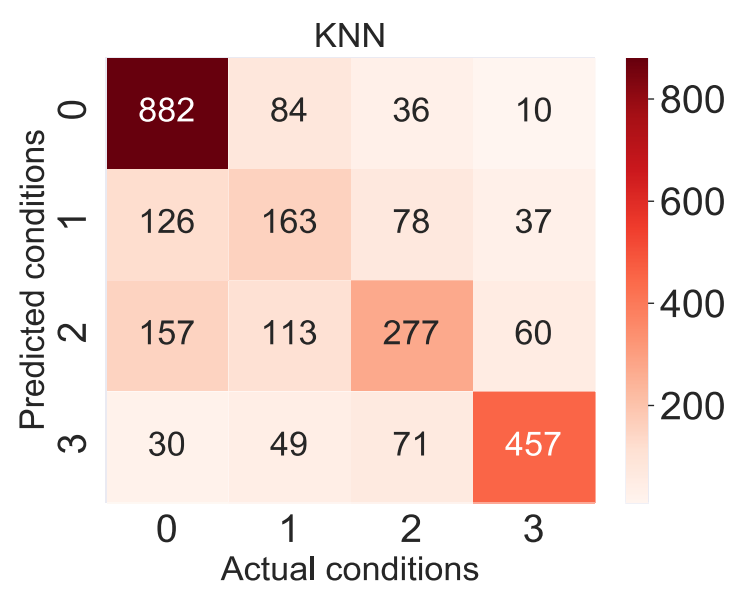

(d) KNN confusion matrix

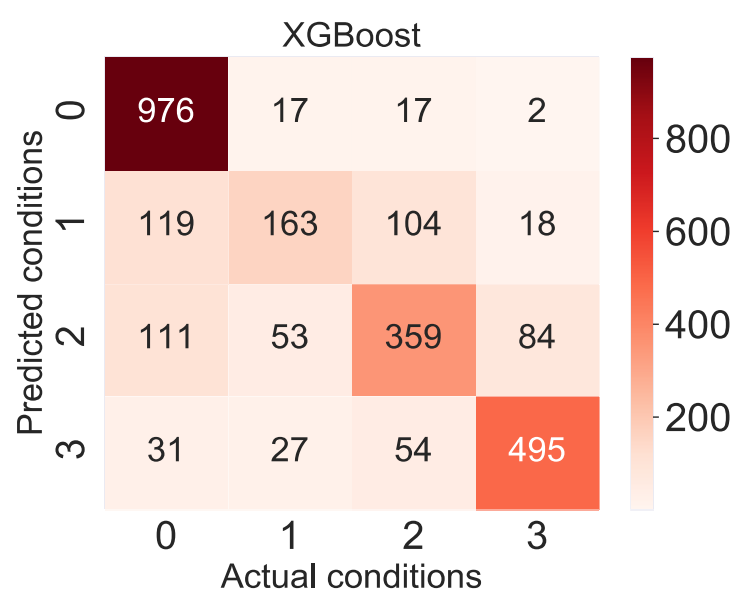

(f) XGBoost confusion matrix

Patterns classification: 0-10\% damaged condition, 1-20\% damaged condition, 3-30\% damaged condition, 4-40\% damaged condition.

Figure 12. Confusion matrix between actual conditions and predicted conditions. 


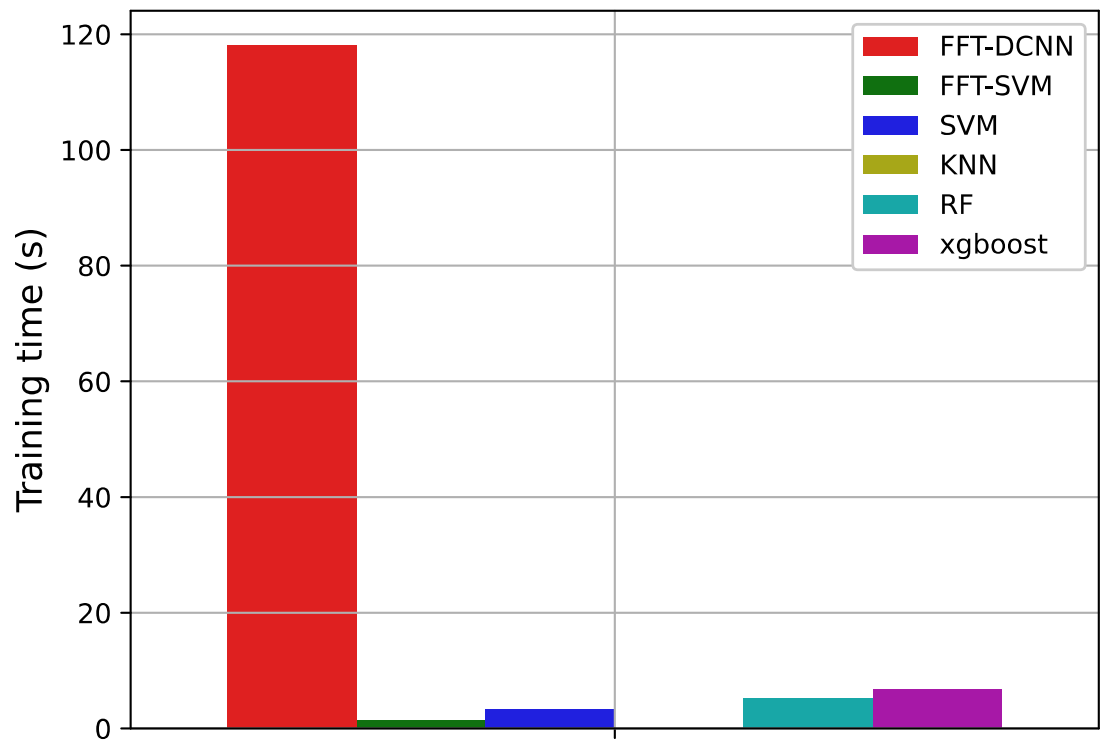

Algorithms

Figure 13. Comparison of training time between the proposed method and ML algorithms.

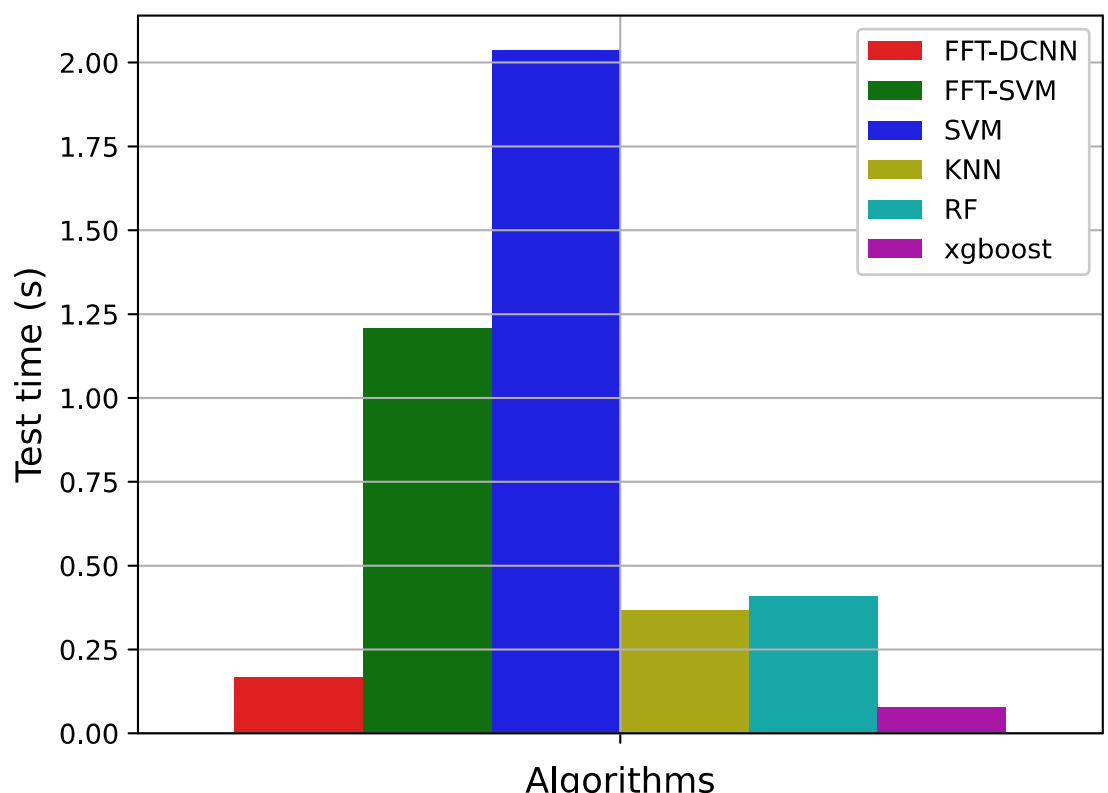

Figure 14. Comparison of test time between the proposed method and ML algorithms.

Table 6. Comparison of cost time between the proposed method and ML algorithms.

\begin{tabular}{ccc}
\hline Method & Training Time (s) & Test Time (s) \\
\hline FFT-DCNN & 118.152 & 0.168 \\
FFT-SVM & 1.384 & 1.207 \\
SVM & 3.391 & 2.038 \\
KNN & 0.056 & 0.368 \\
RF & 5.253 & 0.408 \\
XGBoost & 6.754 & 0.079 \\
\hline
\end{tabular}

Moreover, the test time is an important evaluation metric to judge whether the algorithm can be utilized in actual engineering. It can be seen that the proposed method takes a short 
time on test datasets, compared with FFT-SVM, SVM, KNN, and RF. Thus, the proposed method can be designed for recognizing structural damage in actual civil engineering.

\section{Experimental Results and Discussion for ASCE Benchmark}

\subsection{Data Description and Experimental Setup}

To verify the effectiveness of the proposed method in a complex environment, the experimental phase II of the SHM benchmark is utilized for the study. The datasets and experimental process of the ASCE benchmark were published by the American Society of Civil Engineers (ASCE) SHM task group. The height of the ASCE benchmark is $3.6 \mathrm{~m}$, and the footprint dimensions are $2.5 \mathrm{~m} \times 2.5 \mathrm{~m}$, shown in Figure 15. The experiment aimed to provide a unified test bed for evaluating the ability of structural damage detection using different methods.

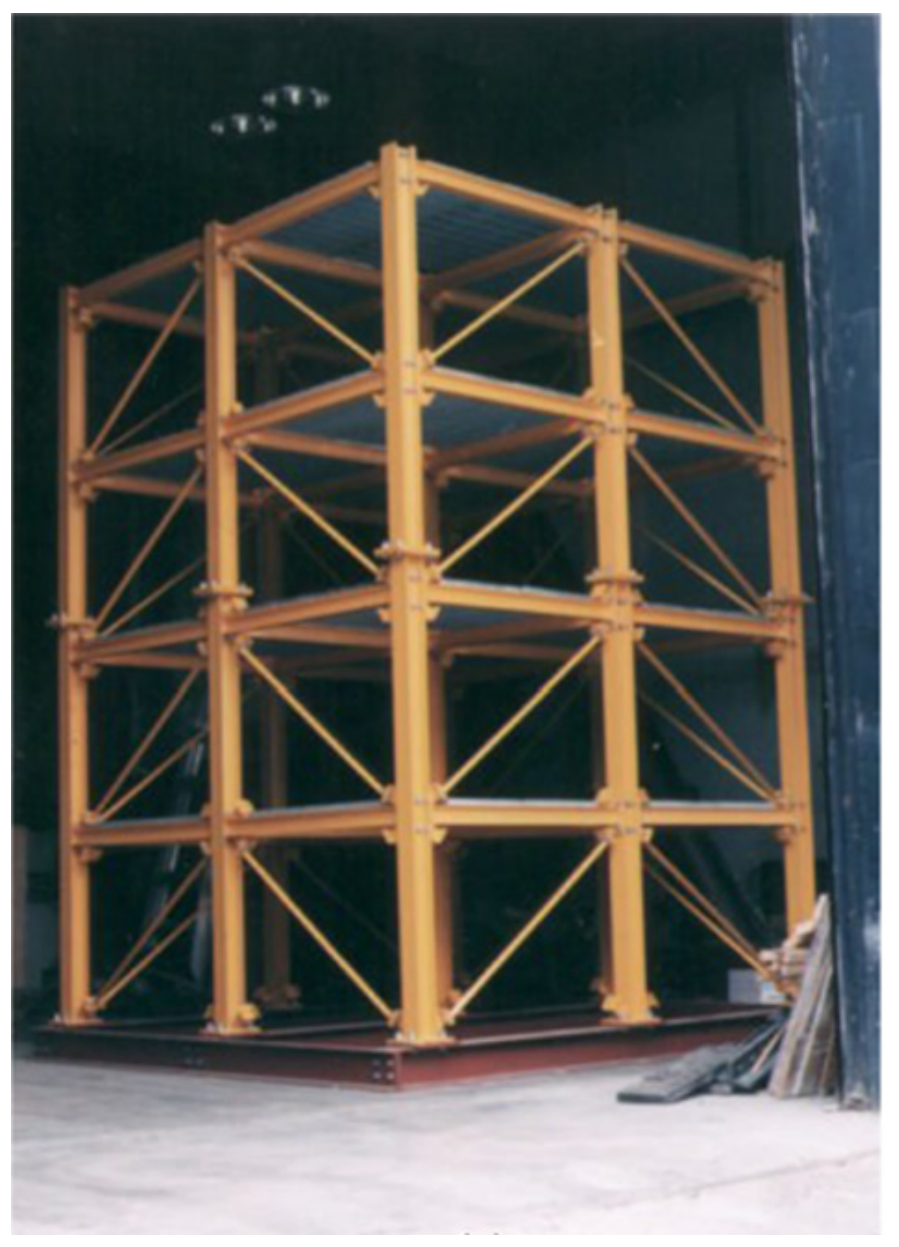

Figure 15. Four-story structure of ASCE benchmark.

The experiment collected acceleration signals from 15 accelerometers under ambient excitation, impact hammer excitation, and $5-50 \mathrm{~Hz}$ randomly generated shaker excitation. Moreover, Case 1 was measured for $120 \mathrm{~s}$, Case 6 was measured for $300 \mathrm{~s}$, and the remaining cases were measured for $360 \mathrm{~s}$. The sampling frequency for all accelerometers were set to $200 \mathrm{~Hz}$. As shown in Table 7 and Figure 16, the damaged degree was increased gradually from undamaged in Case 1 to very damaged in Case 9. Cases 2-7 removed the inclined supports at specific locations to generated structural damage, and Cases 8 and 9 loosed bolts at joint locations. In total, 31,680 samples were acquired by slicing with a window of 200 points during experimental process. Then, the dataset was divided into training, verification, and testing datasets according to the ratio of 6:2:2. That sample number for training, verification, and testing datasets are $19,008,6336$, and 6336, respectively. 
Table 7. Description of the structural cases for the benchmark [30].

\begin{tabular}{cc}
\hline Structural Conditions & Description \\
\hline Case 1 & Undamaged condition \\
Case 2 & Inclined supports located on the first floor was removed \\
Case 3 & Inclined supports located on the first and fourth floors were removed \\
Case 4 & Inclined supports on all floors were removed in one bay \\
Case 5 & All inclined supports were removed on the west face \\
Case 6 & Case 5 + inclined supports located on the second floor were removed \\
Case 7 & All inclined supports on all faces were removed \\
Case 8 & Loosed bolts on first and second floors of the beam + Case 7 \\
Case 9 & Loosed bolts on all floors of the beam on west face + Case 7 \\
\hline
\end{tabular}

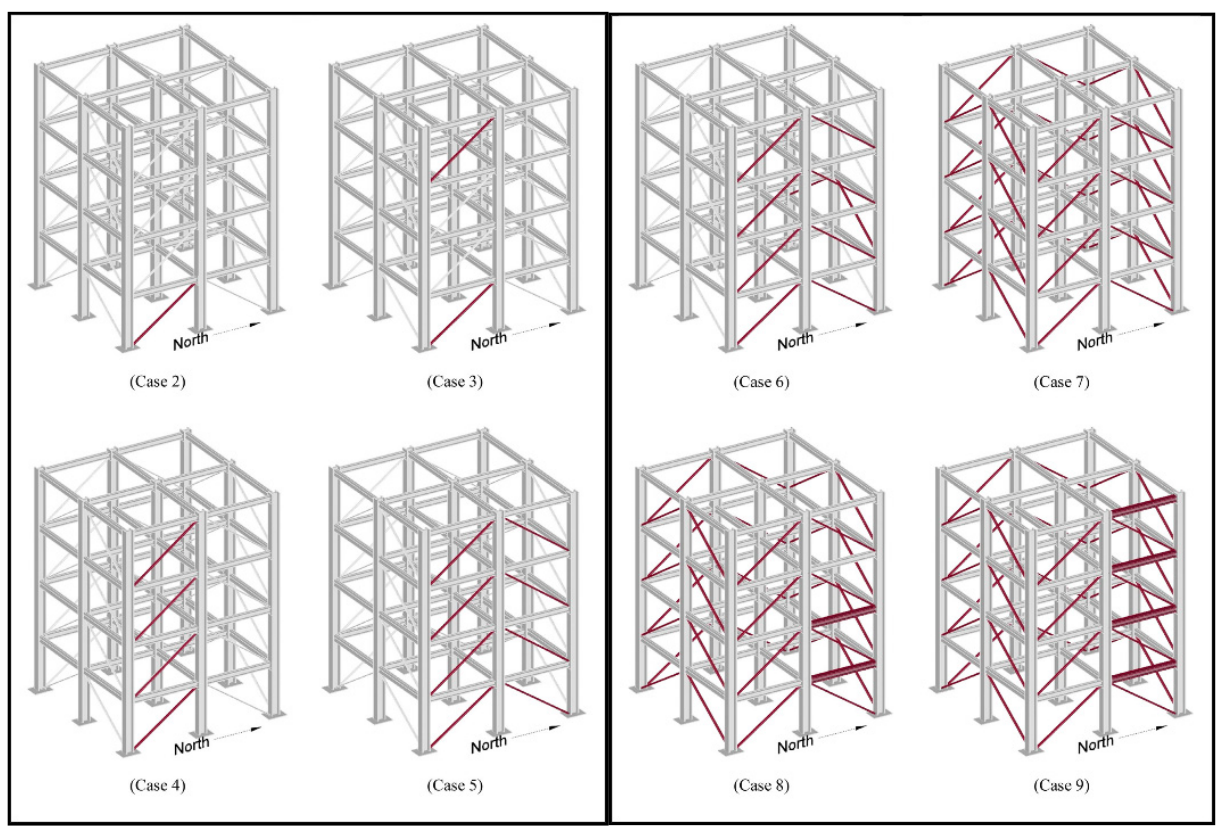

Figure 16. Structural damaged condition 2-9 of ASCE benchmark. (Red color denotes removed inclined supports locations or loosed bolts).

\subsection{FFT-DCNN Testing Result for ASCE Benchmark}

The historical curve of Fold 4 is presented as an example. It can be seen from Figure 17 that there are training, testing, and validation datasets in each epoch. The accuracies of validation and testing reach $80 \%$ after Epoch 250. It indicates that the FFT-DCNN has a faster-trained process and convergence speed.

Four-fold crossvalidation results in the ASCE benchmark dataset are shown in Table 8. In each iteration, the verification accuracy is $88.06 \%$ on average, and the testing accuracy is $87.30 \%$ on average. The experimental result shows that the FFT-DCNN model is suitable for structural damage detection.

Table 8. Accuracy of four-fold cross-validation.

\begin{tabular}{ccc}
\hline Method & Validation (\%) & Testing (\%) \\
\hline Fold-1 & 87.09 & 86.78 \\
Fold-2 & 87.93 & 86.38 \\
Fold-3 & 88.11 & 87.94 \\
Fold-4 & 89.12 & 88.10 \\
Mean & 88.06 & 87.30 \\
\hline
\end{tabular}




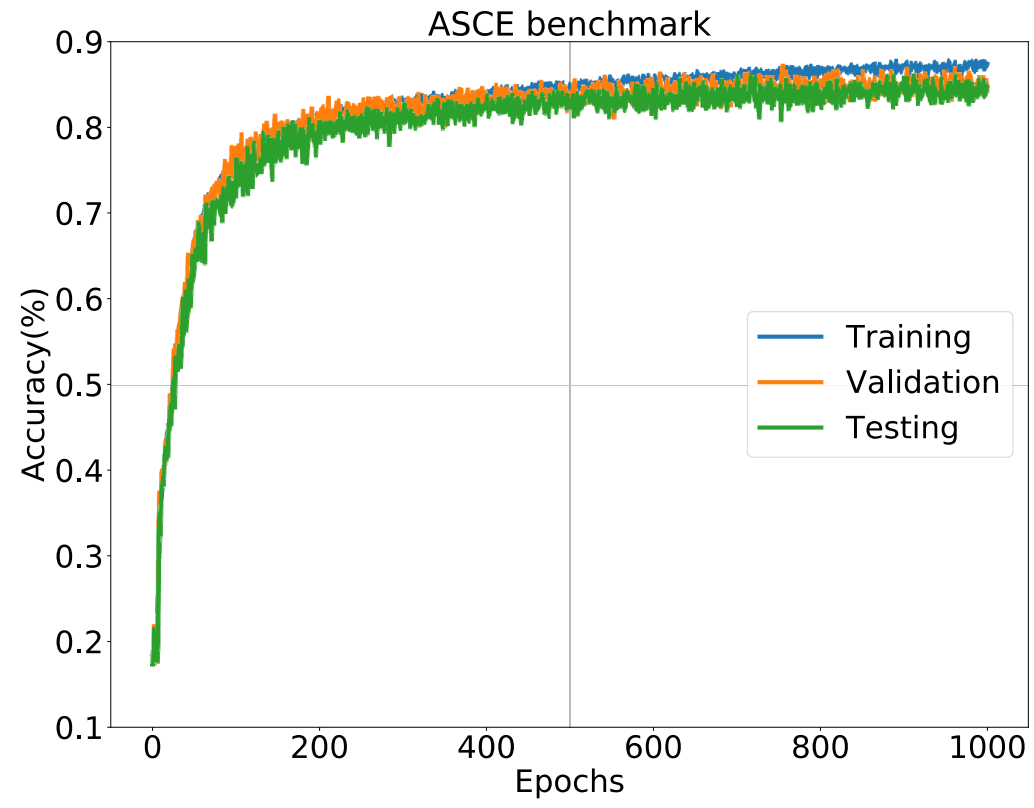

Figure 17. Confusion matrix of damage identification for Fold 4.

Figure 18 denotes the best result of Fold- 4 for the confusion matrix. It can be seen that the label $\mathrm{C} 1$ is undamaged conditions, and the labels $\mathrm{C} 2-\mathrm{C} 9$ denote the damage conditions. The overall identification accuracy of 9 structural damage detection is $88.10 \%$, and the error rate is $11.90 \%$. The accuracy of C2, C6, C7, and C8 is more than $90 \%$. C3, C4, and C9 is $88 \%, 84 \%$, and $89 \%$, respectively. While the $C 1$ and $C 5$ have wrong identification, but its accuracy is still over 70\%, in addition, because the number of samples reaches 31,680 and it is large enough. Thus, the accuracy of FFT-CNN reaches $87.30 \%$ on average, which can satisfy the demand for structural damage detection in actual environment.

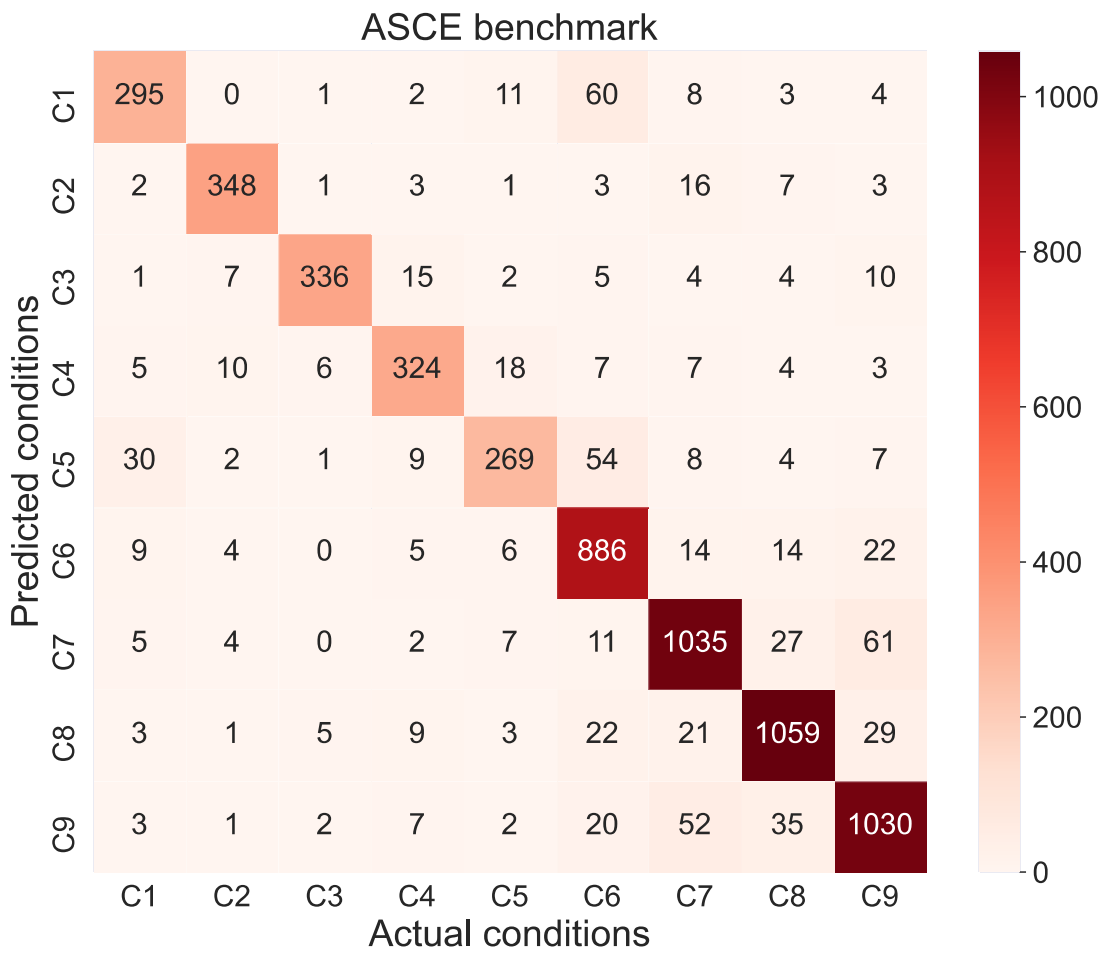

Figure 18. Confusion matrix of damage identification for the Fold-4. 


\subsection{Comparative Analysis for Different Methods}

The classic methods can extract sensitive features from acceleration to recognize structural damage conditions. Table 9 shows the comparison between the proposed methods and classic methods. It indicates that the proposed method achieves higher performance than existing methods, including FFT-SVM, SVM, KNN, RF, and XGBoost. The experimental result shows the proposed method high identification ability in recognizing damaged conditions of civil structure.

Table 9. Comparison result between the proposed method and ML algorithms.

\begin{tabular}{cc}
\hline Method & Accuracy (\%) \\
\hline FFT-DCNN & 87.30 \\
FFT-SVM & 21.29 \\
SVM & 18.19 \\
KNN & 60.57 \\
RF & 71.33 \\
XGBoost & 72.80 \\
\hline
\end{tabular}

\section{Conclusions and Future Work}

This study proposed a novel FFT-DCNN model to identify structural damage detection and was verified on a three-story building structure and ASCE benchmark. The main contributions of this paper are summarized as follows.

(1) A novel data-driven method is proposed by combining FFT with DCNN, effectively handling vibration signals and accurately recognizing structural damage conditions.

(2) Compared with traditional damage detection such as FFT-SVM and SVM, KNN, Random Forest, and XGBoost, the FFT-DCNN model automatically extracts features from the structure under different damage conditions and achieve higher accuracy in $93.38 \%$ for the three-story building structure and accuracy in $87.30 \%$ for the ASCE benchmark.

(3) For time consumption, FFT-DCNN takes a short time on test datasets, compared with FFT-SVM, SVM, KNN, and RF. It indicates that the proposed method can be designed for recognizing structural damage in actual engineering.

(4) This method also has some limitations. Compared with "shallow" ML methods, such as SVM and XGBoost, this method achieves high accuracy relying on a number of training datasets. Thus, we might utilize the transfer learning method to reduce training simple for accurately recognizing structural damage detection in the future.

Author Contributions: Writing-original draft preparation and visualization and writing-review and editing, Y.H.; data curation and investigation, H.C.; visualization, D.L.; methodology and software L.Z. All authors have read and agreed to the published version of the manuscript.

Funding: The work described in this paper was supported by the Scientific Research Program of Chongqing College of Humanities, Science \& Technology (Grant No. CQRKZX2020003), the Science and Technology Research Program of Chongqing Municipal Education Commission (Grant No. KJQN201901801), the Scientific Research Program of Chongqing College of Humanities, Science \& Technology (Grant No.CQRKZX2020004), Foundation project of Scientific Research Platform of Hechuan for Chengdu-Chongqing Economic Area Research Base, and the Teaching Reform Project of School (Grant No. 19CRKXJJG05).

Institutional Review Board Statement: Not applicable.

Informed Consent Statement: Not applicable.

Conflicts of Interest: The authors declare that no conflict interest for the present study was found. 


\section{References}

1. Palma, P.; Steiger, R. Structural Health Monitoring of Timber Structures-Review of Available Methods and Case Studies. Constr. Build. Mater. 2020, 248, 118528. [CrossRef]

2. Rahul, V.; Alokita, S.; Jayakrishna, K.; Kar, V.R.; Rajesh, M.; Thirumalini, S.; Manikandan, M. Structural health monitoring of aerospace composites. In Structural Health Monitoring of Biocomposites, Fibre-Reinforced Composites and Hybrid Composites; Elsevier: Amsterdam, The Netherlands, 2019; pp. 33-52, ISBN 978-0-08-102291-7.

3. Xiang, J.; Yang, Z.; Aguilar, J. Structural Health Monitoring for Mechanical Structures Using Multi-Sensor Data. Int. J. Distrib. Sens. Netw. 2018, 14. [CrossRef]

4. Zhang, Z.; Sun, C. Structural Damage Identification via Physics-Guided Machine Learning: A Methodology Integrating Pattern Recognition with Finite Element Model Updating. Struct. Health Monit. 2021, 20, 1675-1688. [CrossRef]

5. Zhang, Z.; Sun, C. A Numerical Study on Multi-site Damage Identification: A Data-driven Method via Constrained Independent Component Analysis. Struct. Control. Health Monit. 2020, 27, e2583. [CrossRef]

6. Santos, A.; Figueiredo, E.; Silva, M.F.M.; Sales, C.S.; Costa, J.C.W.A. Machine Learning Algorithms for Damage Detection: Kernel-Based Approaches. J. Sound Vib. 2016, 363, 584-599. [CrossRef]

7. Chen, Z.; Tse, K.T.; Kwok, K.C.S.; Kareem, A.; Kim, B. Measurement of Unsteady Aerodynamic Force on a Galloping Prism in a Turbulent Flow: A Hybrid Aeroelastic-Pressure Balance. J. Fluids Struct. 2021, 102, 103232. [CrossRef]

8. Chen, Z.; Fu, X.; Xu, Y.; Li, C.Y.; Kim, B.; Tse, K.T. A Perspective on the Aerodynamics and Aeroelasticity of Tapering: Partial Reattachment. J. Wind Eng. Ind. Aerodyn. 2021, 212, 104590. [CrossRef]

9. Gul, M.; Catbas, F. Structural Health Monitoring and Damage Assessment Using a Novel Time Series Analysis Methodology with Sensor Clustering. J. Sound Vib. 2011, 330, 1196-1210. [CrossRef]

10. Stull, C.; Hemez, F.; Farrar, C. On Assessing the Robustness of Structural Health Monitoring Technologies. Struct. Health Monit. -Int. J. 2012, 11, 1-11. [CrossRef]

11. Seyedpoor, S.M.; Nopour, M.H. A Two-Step Method for Damage Identification in Moment Frame Connections Using Support Vector Machine and Differential Evolution Algorithm. Appl. Soft Comput. 2020, 88, 106008. [CrossRef]

12. Guo, H.Y.; Li, Z.L. Structural Damage Identification Based on Bayesian Theory and Improved Immune Genetic Algorithm. Expert Syst. Appl. 2012, 39, 6426-6434. [CrossRef]

13. Wu, J.; Guo, P.; Cheng, Y.; Zhu, H.; Wang, X.-B.; Shao, X. Ensemble Generalized Multiclass Support-Vector-Machine-Based Health Evaluation of Complex Degradation Systems. IEEE/ASME Trans. Mechatron. 2020, 25, 2230-2240. [CrossRef]

14. Ahmad, M.; Shabbir, S.; Raza, R.A.; Mazzara, M.; Distefano, S.; Khan, A.M. Artifacts of Different Dimension Reduction Methods on Hybrid CNN Feature Hierarchy for Hyperspectral Image Classification. Optik 2021, 246, 167757. [CrossRef]

15. Jia, S.; Jiang, S.; Lin, Z.; Li, N.; Xu, M.; Yu, S. A Survey: Deep Learning for Hyperspectral Image Classification with Few Labeled Samples. Neurocomputing 2021, 448, 179-204. [CrossRef]

16. Lauriola, I.; Lavelli, A.; Aiolli, F. An Introduction to Deep Learning in Natural Language Processing: Models, Techniques, and Tools. Neurocomputing 2021. [CrossRef]

17. Gulgec, N.S.; Takáč, M.; Pakzad, S.N. Convolutional Neural Network Approach for Robust Structural Damage Detection and Localization. J. Comput. Civ. Eng. 2019, 33, 04019005. [CrossRef]

18. Tang, Z.; Chen, Z.; Bao, Y.; Li, H. Convolutional Neural Network-Based Data Anomaly Detection Method Using Multiple Information for Structural Health Monitoring. Struct Control. Health Monit 2019, 26, e2296. [CrossRef]

19. Abdeljaber, O.; Avci, O.; Kiranyaz, M.S.; Boashash, B.; Sodano, H.; Inman, D.J. 1-D CNNs for Structural Damage Detection: Verification on a Structural Health Monitoring Benchmark Data. Neurocomputing 2018, 275, 1308-1317. [CrossRef]

20. Zhang, Y.; Miyamori, Y.; Mikami, S.; Saito, T. Vibration-based Structural State Identification by a 1-dimensional Convolutional Neural Network. Comput.-Aided Civil. Infrastruct. Eng. 2019, 34, 822-839. [CrossRef]

21. Hoshyar, A.N.; Samali, B.; Liyanapathirana, R.; Houshyar, A.N.; Yu, Y. Structural Damage Detection and Localization Using a Hybrid Method and Artificial Intelligence Techniques. Struct. Health Monit. 2020, 19, 1507-1523. [CrossRef]

22. Amini Tehrani, H.; Bakhshi, A.; Akhavat, M. An Effective Approach for Structural Damage Localization in Flexural Members Based on Generalized S-Transform. Sci. Iran. 2017. [CrossRef]

23. Nguyen, T.Q. A Data-Driven Approach to Structural Health Monitoring of Bridge Structures Based on the Discrete Model and FFT-Deep Learning. J. Vib. Eng. Technol. 2021. [CrossRef]

24. Fan, N.; Balan, R.V.; Rosca, J. Comparison of Wavelet-and FFT-Based Single-Channel Speech Signal Noise Reduction Techniques; Truchetet, F., Laligant, O., Eds.; International Society for Optics and Photonics: Philadelphia, PA, USA, 2004; pp. 127-138.

25. Gopinathan, S.; Kokila, R.; Thangavel, P. Wavelet and FFT Based Image Denoising Using Non-Linear Filters. IJECE 2015, 5, 1018. [CrossRef]

26. Zheng, J.; Liao, L.; Chen, Y. Damage Identification Based on Convolutional Neural Network and Recurrence Graph for Beam Bridge. Struct. Health Monit. 2020. [CrossRef]

27. Malek, S.; Melgani, F.; Bazi, Y. One-Dimensional Convolutional Neural Networks for Spectroscopic Signal Regression: Feature Extraction Based on 1D-CNN Is Proposed and Validated. J. Chemom. 2018, 32, e2977. [CrossRef]

28. Hung, D.V.; Hung, H.M.; Anh, P.H.; Thang, N.T. Structural Damage Detection Using Hybrid Deep Learning Algorithm. STCE 2020, 14, 53-64. [CrossRef] 
29. Pan, H.; Gui, G.; Lin, Z.; Yan, C. Deep BBN Learning for Health Assessment toward Decision-Making on Structures under Uncertainties. KSCE J. Civ. Eng. 2018, 22, 928-940. [CrossRef]

30. Dyke, S.J.; Bernal, D.; Beck, J.; Ventura, C. Experimental Phase II of the Structural Health Monitoring Benchmark Problem. Lakartidningen 2003, 105, 2239-2240.

31. Lin, Y.; Nie, Z.; Ma, H. Structural Damage Detection with Automatic Feature-Extraction through Deep Learning: Structural Damage Detection with Automatic Feature-Extraction through Deep Learning. Comput.-Aided Civil. Infrastruct. Eng. 2017, 32, 1025-1046. [CrossRef]

32. Wu, H.; Gu, X. Towards Dropout Training for Convolutional Neural Networks. Neural Netw. 2015, 71, 1-10. [CrossRef]

33. Fan, Z.; Li, C.; Chen, Y.; Mascio, P.D.; Chen, X.; Zhu, G.; Loprencipe, G. Ensemble of Deep Convolutional Neural Networks for Automatic Pavement Crack Detection and Measurement. Coatings 2020, 10, 152. [CrossRef]

34. Gui, G.; Pan, H.; Lin, Z.; Li, Y.; Yuan, Z. Data-Driven Support Vector Machine with Optimization Techniques for Structural Health Monitoring and Damage Detection. KSCE J. Civ. Eng. 2017, 21, 523-534. [CrossRef]

35. Lei, X.; Sun, L.; Xia, Y.; He, T. Vibration-Based Seismic Damage States Evaluation for Regional Concrete Beam Bridges Using Random Forest Method. Sustainability 2020, 12, 5106. [CrossRef]

36. Vitola, J.; Pozo, F.; Tibaduiza, D.; Anaya, M. A Sensor Data Fusion System Based on K-Nearest Neighbor Pattern Classification for Structural Health Monitoring Applications. Sensors 2017, 17, 417. [CrossRef] [PubMed]

37. Leon-Medina, J.X.; Anaya, M.; Parés, N.; Tibaduiza, D.A.; Pozo, F. Structural Damage Classification in a Jacket-Type Wind-Turbine Foundation Using Principal Component Analysis and Extreme Gradient Boosting. Sensors 2021, 21, 2748. [CrossRef] 